\title{
Differentiable spaces that are subcartesian
}

\author{
Richard Cushman and Jędrzej Śniatyck: 1
}

\begin{abstract}
We show that the differential structure of the orbit space of a proper action of a Lie group on an a smooth manifold is continuously reflexive. This implies that the orbit space is a differentiable space in the sense of Smith, which ensures that the orbit space has an exterior algebra of differential forms, which satisfies Smith's version of de Rham's theorem. Because the orbit space is a locally closed subcartesian space, it has vector fields and their flows.
\end{abstract}

\section{Introduction}

The aim of this paper is to show that the differential structure of the orbit space $M / G$ of a proper action of a Lie group $G$ on an a smooth manifold $M$ is continuously reflexive. This implies that $M / G$ a differentiable space in the sense of Smith. This ensures that $M / G$ has an exterior algebra of differential forms, which satisfies Smith's version of de Rham's theorem. Because $M / G$ is a locally closed subcartesian space, it has vector fields and their flows.

The concepts of a differentiable structure and a differentiable space were introduced by Smith in 1966 [13]. They allowed him to define the notions of a differential form and exterior derivative, which he used to prove a version of de Rham's theorem. Smith's paper has not received the attention of mathematical community that it deserves. In section 5 we give a comprehensive review of results in Smith [13].

Subcartesian spaces and differential spaces were introduced in 1967 by Aronszajn [1] and Sikorski [11, respectively. Subcartesian spaces are Hausdorff spaces locally diffeomorphic to arbitrary subsets of $\mathbb{R}^{n}$, where their differential structure is given by an atlas of singular charts. The differential structure of a differential space is given by its ring of smooth functions. In 1973, Walczak [15] showed that subcartesian spaces can be treated as a subcategory of differential spaces. This result allows for a discussion of the geometry of a subcartesian space $S$ in terms of its differential structure $C^{\infty}(S)$. In particular, derivations of $C^{\infty}(S)$ give rise to the notion of vector fields on $S$ and their flows. We summarize the aspects of the theory of subcartesian spaces needed here in section 2 following [14] supplemented by [3].

We introduce the notion of a continuously reflexive differential structure, which is modeled on the notion of a reflexive differential structure discussed

\footnotetext{
${ }^{1}$ version: December 17, 2019

1 Department of Mathematics and Statistics, University of Calgary e-mail: rcushman@ucalgary.ca and sniatycki@ucalgary.ca
} 
in Batubenge et al. [2]. We show that, if the differential structure $C^{\infty}(S)$ of a subcartesian space $S$ is continuously reflexive, then it is a differentiable structure on $S$ in the sense of Smith. This ensures that a subcartesian space $S$ with continuously reflexive differential structure $C^{\infty}(S)$ has an exterior algebra of differential forms, which satisfies Smith's version of de Rham's theorem.

The category of locally closed subcartesian differential spaces whose differential structure is continuously reflexive and smooth maps between them contains the category of orbit spaces of a proper action on a smooth manifold and smooth maps between them. In more detail, let $\Phi$ be a proper action of a Lie group $G$ on a connected smooth manifold $M$. By a result of Palais [10] the $G$-action $\Phi$ has a slice $S_{m}$ at each point $m \in M$. We denote the space of smooth $G$-invariant functions on $M$ by $C^{\infty}(M)^{G}$. Let $M / G=\{G \cdot m \mid m \in M\}$ be the space of $G$-orbits in $M$ with $G$-orbit map $\pi: M \rightarrow M / G: m \mapsto G \cdot m$. Classically it is known that $M / G$ with the quotient topology is a Hausdorff topological manifold, see [5] chpt VII]. The space $C^{\infty}(M / G)=\left\{f: \bar{M} \rightarrow \mathbb{R} \mid \pi^{*} f \in C^{\infty}(M)^{G}\right\}$ is a differential structure on $M / G$, see Cushman and Sniatycki [4]. The differential space topology on $M / G$ is equivalent to the quotient topology, see [14, prop. 4.3.1, p.68]. For each $m \in M$ let $G_{m}=\left\{g \in G \mid \Phi_{g}(m)=m\right\}$. Since the differential spaces $\left(S_{m} / G_{m},\left(C^{\infty}\left(T_{m} S_{m}\right)^{G_{m}}\right)\right.$ and $\left(M / G, C^{\infty}(M)^{G}\right)$ are locally diffeomorphic, see [4, it follows that the differential space $\left(M / G, C^{\infty}(M / G)\right)$ is subcartesian and is locally closed, see [6, lem. 4.6]. Hence $M / G$ is paracompact. In section six we show that the differential structure $C^{\infty}(M / G)$ is continuously reflexive. Thus $M / G$ is a differentiable space in the sense of Smith.

\section{Subcartesian differential spaces}

A differential structure on a topological space $S$ is a family $C^{\infty}(S)$ of real valued functions on $S$ satisfying the following conditions:

1. The collection $\left\{f^{-1}(I) \mid f \in C^{\infty}(S)\right.$ and $I$ is open interval in $\left.\mathbb{R}\right\}$ is a subbasis for the topology of $S$.

2. For every $n \in \mathbb{N}$, if $f_{1}, \ldots, f_{n} \in C^{\infty}(S)$ and $F \in C^{\infty}\left(\mathbb{R}^{n}\right)$, then $F\left(f_{1}, \ldots, f_{n}\right) \in C^{\infty}(S)$.

3. If $f: S \rightarrow \mathbb{R}$ is a function such that for every $x \in S$ there exists an open neighbourhood $U$ of $x$ and a function $f_{x} \in C^{\infty}(S)$ satisfying $\left.f_{x}\right|_{U}=\left.f\right|_{U}$, then $f \in C^{\infty}(S)$. Here, the vertical bar denotes restriction.

A function $f \in C^{\infty}(S)$ is called a smooth function on $S$. It follows from condition 1 , that a smooth function on $S$ is continuous. Condition 2 with $F\left(f_{1}, f_{2}\right)=$ $a f_{1}+b f_{2}$, where $a, b \in \mathbb{R}$ shows that $C^{\infty}(S)$ is a real vector space. Taking $F\left(f_{1}, f_{2}\right)=f_{1} \cdot f_{2}$ shows that $\left(C^{\infty}(S), \cdot\right)$ is a commutative associative algebra with unit under multiplication $\cdot$

In his original definition Sikorski 11 defined $C^{\infty}(S)$ to be a family of functions on $S$ satisfying condition 2 . He then used condition 1 to define a topology 
on $S$. Finally, he imposed condition 3 as a consistency condition.

A topological space $S$ endowed with a differential structure $C^{\infty}(S)$ is called a differential space.

A map $\varphi: R \rightarrow S$ between diferential spaces is smooth if $\varphi^{*} f=f \circ \varphi \in$ $C^{\infty}(R)$ for every $f \in C^{\infty}(S)$. The map $\varphi$ is a diffeomorphism if it is invertible and $\varphi^{-1}$ is smooth.

Proposition 2.1 A smooth map between differential spaces is continuous.

Proof. See proposition 2.1.5 in [14].

An alternative way of constructing a differential structure on a set $S$, used by Sikorski [12], goes as follows. Let $\mathcal{F}$ be a family of real valued functions on $S$. Endow $S$ with the topology generated by the subbasis $\left\{f^{-1}(I) f \in \mathcal{F}\right.$ and $I$ an open interval in $\mathbb{R}\}$. Define $C^{\infty}(S)$ by saying that $h \in C^{\infty}(S)$ if and only if for each $x \in S$ there is an open subset $U$ of $S$, functions $f_{1}, \ldots, f_{n} \in \mathcal{F}$, and $F \in C^{\infty}\left(\mathbb{R}^{n}\right)$ such that $\left.h\right|_{U}=\left.F\left(f_{1}, \ldots, f_{n}\right)\right|_{U}$. Clearly $\mathcal{F} \subseteq C^{\infty}(S)$. Moreover,

Theorem 2.2 The family $C^{\infty}(S)$ is a differential structure on $S$.

Proof. See theorem 2.1.7 in [14].

We refer to $C^{\infty}(S)$ as the differential structure on $S$ generated by the family $C^{\infty}(S)$.

Proposition 2.3 Let $M$ be a smooth manifold. Then $C^{\infty}(M)$ is a differential structure.

Proof. To see this we check that the defining properties of a differential structure hold. First, since every function $f \in C^{\infty}(M)$ is continuous, the set $\left\{f^{-1}(I) \subseteq M \mid f \in C^{\infty}(M)\right.$ and $I$ an open interval in $\left.\mathbb{R}\right\}$ is a subbasis for the manifold topology of $M$. Second, for every $n \in \mathbb{N}$ with $f_{1}, \ldots, f_{n} \in C^{\infty}(M)$ and $F \in C^{\infty}\left(\mathbb{R}^{n}\right)$ we see that $F\left(f_{1}, \ldots, f_{n}\right) \in C^{\infty}(M)$. Third, suppose that $f$ is a function on $M$ such that for every $m \in M$ there is an open neighborhood $U$ of $m$ in $M$ and a function $f_{m} \in C^{\infty}(M)$ such that $\left.f\right|_{U}=\left.f_{m}\right|_{U}$. Choosing $U$ to be the domain of a chart of $M$ at $m$, it follows that $f$ is a smooth function on $M$. Consequently, $C^{\infty}(M)$ is a differential structure on $M$.

Let $R$ be a differential space with differential structure $C^{\infty}(R)$. Let $S$ be a subset of $R$ endowed with the subspace topology, in which open subsets of $S$ are of the form $S \cap U$, where $U$ is an open subset of $R$. Let $C^{\infty}(R)$ be the differential structure generated by the restriction to $R$ of smooth functions on $S$. We say that the differential space $\left(S, C^{\infty}(S)\right)$ is a differential subspace of the differential space $\left(R, C^{\infty}(R)\right)$.

Proposition 2.4 The space $\left.C^{\infty}(R)\right|_{S}$ of restrictions to $S \subseteq R$ of smooth functions on $R$ generates a differential structure $C^{\infty}(S)$ on $S$ such that the differential space topology on $S$ coincides with its subspace topology. The inclusion map $\iota: S \rightarrow R$ is smooth.

Proof. See proposition 2.1.8 in [14]. 
We can characterize a smooth $n$-dimensional Hausdorff manifold as a Hausdorff differential space $S$ such that every point $x \in S$ has a neighborhood diffeomorphic to an open subset $V$ of $\mathbb{R}^{n}$. Here the differential structures on $U$ and $V$ are generated by the restriction of smooth functions on $S$ to $U$ and on $\mathbb{R}^{n}$ to $V$, respectively.

We weaken the above definition of manifold as follows. A differential space $\left(S, C^{\infty}(S)\right)$ is subcartesian if its topology is Hausdorff and for every point $x \in S$ there is an open neighborhood $U$, which is diffeomorphic to a subset $V$ of some $\mathbb{R}^{n}$. Observe that $V$ is an arbitrary subset of $\mathbb{R}^{n}$ and that $n$ may depend on $x \in S$. More precisely, a differential space $\left(S, C^{\infty}(S)\right)$ is subcartesian if and only if

1. the topology on $S$ defined by its differential structure $C^{\infty}(S)$ is Hausdorff;

2. for every $x \in S$ there is an open subset $U_{x}$ of $S$ containing $x$ and $^{2}$ a diffeomorphism $\varphi_{x}:\left(U_{x}, C^{\infty}\left(U_{x}\right)\right) \rightarrow\left(V_{x}=\varphi_{x}\left(U_{x}\right), C^{\infty}\left(V_{x}\right)\right)$ of differential spaces, where $V_{x} \subseteq \mathbb{R}^{n}, \iota_{V_{x}}: V_{x} \rightarrow \mathbb{R}^{n}$ is the inclusion map and $C^{\infty}\left(V_{x}\right)=\iota_{V_{x}}^{*}\left(C^{\infty}\left(\mathbb{R}^{n}\right)\right)$.

A diffeomorphism of an open subset of $S$ onto a subset of some $\mathbb{R}^{n}$ is called a chart on $S$. The family of all charts is the complete atlas on $S$. An example of a subcartesian differential space is an arbitrary subset $W$ of Euclidean space $\mathbb{R}^{n}$ with subset topology and differential structure $\left.C^{\infty}\left(\mathbb{R}^{n}\right)\right|_{W}$ consisting of restrictions of smooth functions on $\mathbb{R}^{n}$ to $W$. We refer to a differential space, which is subcartesian, as a subcartesian differential space, because subcartesian spaces with charts is an active area of research.

\section{Vector fields on a subcartesian space}

\subsection{Tangent bundle}

Let $\left(S, C^{\infty}(S)\right)$ be a differential space. A derivation of $C^{\infty}(S)$ at $x \in S$ is a real linear mapping $v_{x}: C^{\infty}(S) \rightarrow \mathbb{R}$ such that

$$
v_{x}\left(f_{1} f_{2}\right)=v_{x}\left(f_{1}\right) f_{2}(x)+f_{1}(x) v_{x}\left(f_{2}\right) \text { for every } f_{1}, f_{2} \in C^{\infty}(S) .
$$

Let $T_{x} S$, the tangent space to $S$ at $x$, be the set of all derivations of $C^{\infty}(S)$ at $x$. Let $T S=\left\{v=\left(x, v_{x}\right) \mid x \in S \& v_{x} \in T_{x} S\right\}$ be the tangent bundle of $S$ with bundle projection map $\tau_{S}: T S \rightarrow S: v=\left(x, v_{x}\right) \mapsto x$, which assigns to every derivation $v_{x} \in T_{x} S$ at $x$ the point $x$. The differential structure $C^{\infty}(T S)$ on $T S$ is generated by the family of functions $\left\{\tau_{S}^{*} f, \mathrm{~d} f \mid f \in C^{\infty}(S)\right\}$, where $\mathrm{d} f: T S \rightarrow \mathbb{R}: v=\left(x, v_{x}\right) \mapsto v_{x}(f)$. The tangent bundle projection map $\tau_{S}:\left(T S, C^{\infty}(T S)\right) \rightarrow\left(S, C^{\infty}(S)\right)$ is a smooth map of differential spaces.

Lemma 3.1.1 If $\varphi: S \rightarrow R$ is a smooth map of differential spaces, then

$$
T \varphi: T S \rightarrow T R: v=\left(x, v_{x}\right) \mapsto\left(\varphi(x), T_{x} \varphi\left(v_{x}\right)\right),
$$

\footnotetext{
${ }^{2}$ In other words, $f \in C^{\infty}\left(V_{x}\right)$ if and only if there is an $F \in C^{\infty}\left(\mathbb{R}^{n}\right)$ such that $\left.F\right|_{V_{x}}=f$.
} 
where $\left(T_{x} \varphi\left(v_{x}\right)\right) f=v_{x}\left(\varphi^{*} f\right)$ for every $f \in C^{\infty}(R)$, is a smooth map of differential spaces.

Proof. It suffices to show that $(T \varphi)^{*} \mathcal{F}_{R} \subseteq \mathcal{F}_{S}$, where $\mathcal{F}_{R}=\left\{\tau_{R}^{*} f, \mathrm{~d} f \mid f \in\right.$ $\left.C^{\infty}(R)\right\}$ and $\mathcal{F}_{S}=\left\{\tau_{S}^{*} g, \mathrm{~d} g \mid g \in C^{\infty}(S)\right\}$. Since $T \varphi$ sends $T_{x} S$ to $T_{\varphi(x)} R$ for every $x \in S$, we have $\tau_{R} \circ T \varphi=\varphi \circ \tau_{S}$. So for every $f \in C^{\infty}(R)$ we get

$$
(T \varphi)^{*}\left(\tau_{R}^{*} f\right)=\left(\tau_{R} \circ T \varphi\right)^{*} f=\left(\varphi \circ \tau_{S}\right)^{*} f=\tau_{S}^{*}\left(\varphi^{*} f\right)=\tau_{S}^{*}(g),
$$

for $g=\varphi^{*} f \in C^{\infty}(S)$. And for every $v=\left(x, v_{x}\right) \in T S$ we have

$$
(T \varphi)^{*}(\mathrm{~d} f)(v)=\left(T_{x} \varphi\left(v_{x}\right)(f)\right)(x)=\left(v_{\varphi(x)}\left(\varphi^{*} f\right)\right)(x)=\mathrm{d}\left(\varphi^{*} f\right)(v),
$$

that is, $(T \varphi)^{*}(\mathrm{~d} f)=\mathrm{d}\left(\varphi^{*} f\right)=\mathrm{d} g$, for $g=\varphi^{*} f \in C^{\infty}(S)$. Consequently, $(T \varphi)^{*} \mathcal{F}_{R} \subseteq \mathcal{F}_{S}$, as desired.

Corollary 3.1.1A Suppose that $\varphi: R \rightarrow S$ and $\psi: S \rightarrow U$ are smooth maps of differential spaces, then $T(\psi \circ \varphi)=T \psi \circ T \varphi$.

Proof. Let $v_{x} \in T_{x} R$. Then $v_{\varphi(x)}=T_{x} \varphi\left(v_{x}\right) \in T_{\varphi(x)} S$. Also $v_{\psi(\varphi(x))}=$ $T_{\varphi(x)} \psi\left(v_{\varphi(x)}\right) \in T_{\psi(\varphi(x))} U$. So $T_{\psi(\varphi(x))} \psi \circ T_{\varphi(x)} \varphi$ sends $v_{x}$ to $v_{(\psi \circ \varphi)(x)}$, that is, $T_{\psi(\varphi(x))} \psi \circ T_{\varphi(x)} \varphi=T_{x}(\psi \circ \varphi)$ for every $x \in R$. Thus $T(\psi \circ \varphi)=T \psi \circ T \varphi$.

Corollary 3.1.1B If $\varphi: S \rightarrow R$ is a diffeomorphism of differential spaces, then $T \varphi: T S \rightarrow T R$ is a diffeomorphism of differential spaces.

Proof. This follows immediately because $(T \varphi)^{-1}=T \varphi^{-1}$.

Corollary 3.1.1C If $\left(R, C^{\infty}(R)\right)$ is a differential subspace of $\left(S, C^{\infty}(S)\right)$, then $\left(T R, C^{\infty}(R)\right)$ is a differential subspace of $\left(T S, C^{\infty}(T S)\right)$. In particular, $T R \subseteq$ $T S$.

Proof. Because $R$ is a differential subspace of $S$, the inclusion map $\iota_{R}: R \rightarrow S$ is a smooth map. Hence its tangent

$$
T \iota_{R}: T R \rightarrow T S:\left(x, v_{x}\right) \mapsto\left(y, v_{y}\right)=\left(\iota_{R}(x),\left(T_{x} \iota_{R}\right) v_{x}\right)
$$

is a smooth map. $T \iota_{R}$ is the inclusion map because $T_{x} \iota_{R}: T_{x} R \rightarrow T_{\iota_{R}(x)} S$ is the inclusion map for every $x \in R$. Hence $\left(T R, C^{\infty}(T R)\right)$ is a differential subspace of $\left(T S, C^{\infty}(T S)\right)$. In particular, $T R \subseteq T S$.

Proposition 3.1.2 If $\left(S, C^{\infty}(S)\right)$ is a subcartesian differential space, then its tangent bundle $\left(T S, C^{\infty}(T S)\right)$ is a subcartesian differential space.

Proof. It suffices to show that the smooth functions in $\mathcal{F}=\left\{\tau_{S}^{*} f, \mathrm{~d} f \mid f \in\right.$ $\left.C^{\infty}(S)\right\}$ separate points of $T S$. Let $v=\left(x, v_{x}\right)$ and $w=\left(y, w_{y}\right)$ be two distinct points in $T S$. Suppose that $x=\tau_{S}(v) \neq \tau_{S}(w)=y$. Since $S$ is subcartesian, the topology on $S$ determined by $C^{\infty}(S)$ is Hausdorff. So there are disjoint open neighborhoods $V$ of $x$ and $W$ of $y$ in $S$. Since the bundle projection map $\tau_{S}: T S \rightarrow S$ is smooth, it is continuous. Hence $\tau_{S}^{-1}(V)$ and $\tau_{S}^{-1}(W)$ are open neighborhoods in $T S$ of $v$ and $w$, respectively. Also $\tau_{S}^{-1}(V) \cap \tau_{S}^{-1}(W)=\varnothing$, for if $u \in \tau_{S}^{-1}(V) \cap \tau_{S}^{-1}(W)$, then $\tau_{S}(u) \in V \cap W$, which is a contradiction. Now 
suppose that $x=y$. Since $v \neq w$, it follows that $v_{x} \neq w_{x}$. Thus for some $f \in C^{\infty}(S)$ we have

$$
a=\mathrm{d} f(v)=\mathrm{d} f(x) v_{x}=v_{x}(f) \neq w_{x}(f)=\mathrm{d} f(x) w_{x}=\mathrm{d} f(w)=b,
$$

where $\mathrm{d} f(x): T_{x} S \rightarrow \mathbb{R}: v_{x} \mapsto v_{x}(f)$. Since either $a$ or $b$ is nonzero, the linear map $\mathrm{d} f(x)$ is nonzero and hence is surjective. Consequently, the map $\mathrm{d} f: T S \rightarrow \mathbb{R}$ is surjective. Suppose that $a<b$. The case when $b<a$ is handled similarly and is omitted. We can choose $c<d$ so that $a<c<d<b$. For example let $c=a+\frac{1}{4}(b-a)$ and $d=b-\frac{1}{4}(b-a)$. Let $V=(\mathrm{d} f)^{-1}(-\infty, c)$ and $W=(\mathrm{d} f)^{-1}(d, \infty)$. Then $V$ and $W$ are open subsets of $T S$, since $\mathrm{d} f$ is a smooth, and hence a continuous, function on $T S$. Moreover, $V \cap W=\varnothing$, because $(-\infty, c) \cap(d, \infty)=\varnothing$. Also $v \in V$ and $w \in W$, since $\mathrm{d} f(v)=\mathrm{d} f(x) v_{x}=$ $a \in(-\infty, c)$ and $\mathrm{d} f(w)=\mathrm{d} f(x) w_{x}=b \in(d, \infty)$. Thus the topology on TS generated by the functions in $\mathcal{F}$, and hence in $C^{\infty}(T S)$, is Hausdorff.

A section of the tangent bundle projection map $\tau_{S}: T S \rightarrow S$ is a smooth map $\xi: S \rightarrow T S$ of differential spaces such that $\tau_{S} \circ \xi=\mathrm{id}_{S}$. A real linear map $X: C^{\infty}(S) \rightarrow C^{\infty}(S)$ is a derivation on the differential space $\left(S, C^{\infty}(S)\right)$ if it satisfies Leibniz's rule

$$
X\left(f_{1} f_{2}\right)=X\left(f_{1}\right) f_{2}+f_{1} X\left(f_{2}\right) \text { for every } f_{1}, f_{2} \in C^{\infty}(S) .
$$

Proposition 3.1.3 Every derivation $X$ of $C^{\infty}(S)$ defines a section $X: S \rightarrow$ $T S: x \mapsto X(x)$ of $\tau_{S}$, where $X(x) f=(X f)(x)$ for every $f \in C^{\infty}(S)$.

Proof. See [3, prop. 6].

\subsection{Integral curves of derivations}

Let $c: I \subseteq \mathbb{R} \rightarrow S$ be a smooth map of an interval $I$ containing 0 into the differential space $S$. In other words, $c$ is a smooth map of the differential space $\left(I, C^{\infty}(I)\right)$ into the differential space $\left(S, C^{\infty}(S)\right)$ with $0 \in I$. We say that $c$ is an integral curve of the derivation $X$ of $C^{\infty}(S)$ starting at $x_{0} \in S$ if $c(0)=x_{0}$ and

$$
\frac{\mathrm{d}}{\mathrm{d} t} f(c(t))=X(f)(c(t)) \quad \text { for every } f \in C^{\infty}(S) \text { and every } t \in I .
$$

So $c: I \subseteq \mathbb{R} \rightarrow S$ is an integral curve of $X$ if $(T c)(t)=(X \circ c)(t)$. The following example shows that it is convenient to allow the interval $I$ to be a single point.

Example 3.2.1 Let $\mathbb{Q}$ be the set of rational numbers in $\mathbb{R}$. Since $\mathbb{Q}$ is dense in $\mathbb{R}$, every derivation of $C^{\infty}(\mathbb{R})$ induces a derivation of $C^{\infty}(\mathbb{Q})$. Let $X$ be the derivation of $C^{\infty}(\mathbb{Q})$ induced by the derivation $\frac{\mathrm{d}}{\mathrm{d} x}$ of $C^{\infty}(\mathbb{R})$. In other words, for every $f \in C^{\infty}(\mathbb{Q})$ and every $x_{0} \in \mathbb{Q}$

$$
(X f)\left(x_{0}\right)=\lim _{x \rightarrow x_{0}} \frac{f(x)-f\left(x_{0}\right)}{x-x_{0}},
$$


where the limit is taken over $x \in \mathbb{Q}$. Since no two distinct points in $Q$ can be joined by a continuous curve in $\mathbb{Q}$, it follows that for each $x_{0} \in \mathbb{Q}$ the tangent vector $X\left(x_{0}\right) \in T_{x_{0}} \mathbb{Q}$ is the maximal integral curve of $X$ through $x_{0}$.

Let $\left(S, C^{\infty}(S)\right)$ be a differential space. Let $X$ be a derivation of $C^{\infty}(S)$. A lifted integral curve of $X$ starting at $x_{0} \in S$ is a map $\gamma: I \subseteq \mathbb{R} \rightarrow T S$ such that $\gamma(0)=X\left(x_{0}\right)$ and

$$
\frac{\mathrm{d}}{\mathrm{d} t} f\left(\tau_{S}(\gamma(t))\right)=X(f)\left(\tau_{S}(\gamma(t))\right)
$$

for every $f \in C^{\infty}(S)$ and every $t \in I$ provided that $I \neq\{0\}$.

The following example shows that quite ordinary differential spaces can have a derivation with an integral curve whose domain is a single point.

Example 3.2.2 Consider the function $h(x)=\left\{\begin{array}{c}\mathrm{e}^{\left(-x^{-2}\right)} \\ 0,\end{array}, \begin{array}{l}\text { if } x \neq 0 \\ \text { if } x=0 .\end{array}\right.$. Then $h$ is smooth and it and all of its derivatives vanish at $x=0$. Let

$$
S=\left\{(x, y) \in \mathbb{R}^{2} \mid H(x, y)=y^{2}-h(x) y=0\right\} .
$$

The point $(0,0)$ is an isolated singular point of $S$, since $\mathrm{d} H(x, y)=\left(-h^{\prime}(x) y, 2 y-\right.$ $h(x))$ is equal to $(0,0)$ only when $(x, y)=(0,0)$.

Consider the map $X=\frac{\partial}{\partial x}+v(x) \frac{\partial}{\partial y}$ of $C^{\infty}\left(\mathbb{R}^{2}\right)$ into $C^{0}\left(\mathbb{R}^{2}\right)$, where we have $v(x)=\left\{\begin{array}{cc}0, & \text { if } x \leq 0 \\ h^{\prime}(x), & \text { if } x>0 .\end{array}\right.$. If $F \in C^{\infty}\left(\mathbb{R}^{2}\right)$ then

$$
G(x, y)=X(F)(x, y)=\left\{\begin{array}{cc}
\frac{\partial F}{\partial x}(x, y), & \text { if } x \leq 0 \\
\frac{\partial F}{\partial x}(x, y)+h^{\prime}(x) \frac{\partial F}{\partial y}(x, y), & \text { if } x>0 .
\end{array}\right.
$$

So

$$
\frac{\partial^{n+m} G}{\partial x^{n} \partial y^{m}}(x, y)=\left\{\begin{array}{cc}
\frac{\partial^{n+m+1} F}{\partial x^{n+1} \partial y^{m}}(x, y), & \text { if } x \leq 0 \\
\frac{\partial^{n+m+1} F}{\partial x^{n+1} \partial y^{m}}(x, y)+\sum_{k=0}^{n}\left(\begin{array}{l}
n \\
k
\end{array}\right) h^{(n-k+1)}(x) \frac{\partial^{m+k+1} F}{\partial x^{k} \partial y^{m+1}}(x, y), & \text { if } x>0 .
\end{array}\right.
$$

For all $n \in \mathbb{Z}_{\geq 0}$ we have

$$
\lim _{x \rightarrow 0} \frac{\partial^{n+m} G}{\partial x^{n} \partial y^{m}}(x, 0)=\frac{\partial^{n+m+1} F}{\partial x^{n} \partial y^{m}}(0,0) .
$$

Since all the partial derivatives of $G$ are continuous at $(0,0)$, it follows that $G \in C^{\infty}\left(\mathbb{R}^{2}\right)$. So $X$ is a derivation on $S$. Note that by definition $X(0,0) \neq(0,0)$. The derivation $X$ on $S$ is a vector field on $S_{\text {reg }}=S \backslash\{(0,0)\}$, which is a smooth manifold.

We now show that a maximal integral curve of $X$ starting at $(0,0)$ has domain $\{(0,(0,0)) \in \mathbb{R} \times S\}$. Consider the curve

$$
c: \mathbb{R}_{\neq 0} \rightarrow S_{\text {reg }}: x \mapsto(x, y(x))=\left(x, \mathrm{e}^{-\left(x^{-2}\right)}\right) .
$$


Because $X$ is a vector field on $S_{\text {reg }}$, we see that $c$ is the image of an integral curve of $X$ starting at $\left(1, \mathrm{e}^{-1}\right)$. Now

$$
\gamma: \mathbb{R}_{\neq 0} \rightarrow S: t \mapsto\left(t+1, \mathrm{e}^{-(t+1)^{-2}}\right)=(x(t), y(t))
$$

satisfies $\dot{x}(t)=1$ and $\dot{y}(t)=2 y(t) x(t)^{-3}$, since

$$
\frac{\mathrm{d} y}{\mathrm{~d} t}(t)=\frac{\mathrm{d}}{\mathrm{d} t} \mathrm{e}^{-(t+1)^{-2}}=2(t+1)^{-3} \mathrm{e}^{-(t+1)^{-2}}=2 x(t)^{-3} y(t) .
$$

Consequently, $\gamma$ is an integral curve of the vector field $Z=x^{3} \frac{\partial}{\partial x}+2 y \frac{\partial}{\partial y}$ on $\mathbb{R}^{2} \backslash\{(0,0)\}$. The integral curves of $Z$ satisfy $\frac{\mathrm{d} x}{\mathrm{~d} t}=x^{3}$ and $\frac{\mathrm{d} y}{\mathrm{~d} t}=2 y$. Separating variables gives $\frac{\mathrm{d} x}{x^{3}}=\mathrm{d} t$ and $\frac{\mathrm{d} y}{2 y}=\mathrm{d} t$, which integrates to give the curve

$$
\gamma:(-\infty, 0] \rightarrow S_{\mathrm{reg}}: t \mapsto(x(t), y(t))=\left(\left(\frac{-1}{2 t-1}\right)^{1 / 2}, \mathrm{e}^{(2 t-1)}\right),
$$

that starts at $\left(1, \mathrm{e}^{-1}\right)$ and has the same image as the curve $x \mapsto\left(x, \mathrm{e}^{-\left(x^{-2}\right)}\right)$ when $0<x \leq 1$. Note that $\lim _{t \searrow-\infty} \gamma(t)=(0,0)$. So $\gamma$ is the maximal nonpositive

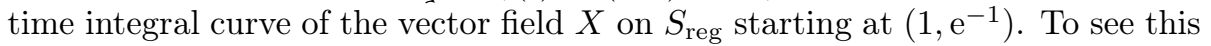
suppose that the domain of a maximal nonpositive time integral curve $\Gamma$ of the derivation $X$ on $S$ starting at $(0,0)$ is an interval $(-\varepsilon, 0]$ for some $\varepsilon>0$. Then there is $t_{0} \in(-\varepsilon, 0) \cap(-\infty, 0)$ such that $\Gamma\left(t_{0}\right)=\gamma\left(t_{0}\right) \in S_{\text {reg. }}$. By uniqueness of integral curves of vector fields $\Gamma=\gamma$ on $(-\varepsilon, 0)$. Hence $\Gamma$ is an extension of the maximal nonpositive time integral curve $\gamma$, which is a contradiction. So $\Gamma$ is only defined on $[0, \infty)$. Repeating the above argument for the integral curve $\gamma^{\vee}:[0, \infty) \rightarrow S_{\text {reg }}: t \mapsto\left(-1+t, \mathrm{e}^{-(-1+t)^{-2}}\right)$ of the vector field $X$ starting at $\left(-1, \mathrm{e}^{-1}\right)$ shows that $\gamma^{\vee}$ is a maximal nonnegative time integral curve of $X$ because $\lim _{t} \nearrow_{\infty} \gamma^{\vee}(t)=(0,0)$. Hence $\Gamma$ is only defined at $t=0$. In other words, the nonzero derivation $X(0,0)=\left.\frac{\partial}{\partial x}\right|_{(0,0)}$ at the point $(0,0)$ is the lifted integral curve of the derivation $X$ on $S$ at $(0,0)$, which has a domain consisting only of $\{(0,(0,0)) \in \mathbb{R} \times S\}$.

Theorem 3.2.3 Let $\left(S, C^{\infty}(S)\right)$ be a subcartesian differential space and let $X$ be a derivation of $C^{\infty}(S)$. For every $x \in S$ there is a unique maximal lifted integral curve of $X$ starting at $x$.

Proof. See [3, thm 8].

\subsection{Vector fields}

A vector field on a smooth manifold $M$ is not only a derivation of $C^{\infty}(M)$ but it has a unique integral curve starting at $m \in M$ and also it generates a local oneparameter group of local diffeomorphisms of $M$. On a subcartesian differential space $\left(S, C^{\infty}(S)\right)$ not all derivations of $C^{\infty}(S)$ generate a local one-parameter group of diffeomorphisms of $S$, see example 3.3.2. We define a vector field on a subcartesian space $\left(S, C^{\infty}(S)\right)$ to be a derivation $X$ of $C^{\infty}(S)$ such that for 
every $x_{0} \in S$ the following conditions hold: 1) there is a unique lifted integral curve of $X$ starting at $x_{0}$, and 2) there is an open neighborhood $U_{x_{0}}$ of $x_{0}$ and an $\varepsilon_{x_{0}}>0$ such that for every $x \in U_{x_{0}}$ the interval $\left(-\varepsilon_{x_{0}}, \varepsilon_{x_{0}}\right)$ is contained in the domain $I_{x}$ of the lifted integral curve $\gamma_{x}: I_{x} \subseteq \mathbb{R} \rightarrow T S$ starting at $x$ of the derivation $X$ and the map

$$
\mathrm{e}^{t X}: U_{x_{0}} \subseteq S \rightarrow S: x \mapsto\left(\tau_{S} \circ \gamma_{x}\right)(t)
$$

is defined for every $t \in\left(-\varepsilon_{x_{0}}, \varepsilon_{x_{0}}\right)$ and is a diffeomorphism of $U_{x_{0}}$ onto the open subset $\mathrm{e}^{t X}\left(U_{x_{0}}\right)$ of $S$.

Recall that a subset $A$ of a topological space $\mathcal{T}$ is locally closed if for every point $x \in A$ there is an open set $U_{x} \in \mathcal{T}$ containing $x$ such that $A \cap U_{x}$ is closed in $U_{x}$. For a locally closed differential space we have a much simpler criterion for recognizing whether a derivation is a vector field.

Proposition 3.3.1 Let $S$ be a locally closed subcartesian differential space. A derivation $X$ of $C^{\infty}(S)$ with unique integral curves is a vector field on $S$ if the domain of every maximal integral curve of $X$ is an open interval in $\mathbb{R}$ which contains 0 .

Proof. See [14, prop 3.2.3, p.35].

The hypothesis that $S$ is locally closed is necessary as the following example shows.

Example 3.3.2 Consider the subset of $\mathbb{R}^{2}$ given by

$$
S=\left\{x=\left(x_{1}, x_{2}\right) \in \mathbb{R}^{2} \mid x_{1}^{2}+\left(1-x_{2}\right)^{2}<1 \text { or } x_{2}=0\right\} .
$$

Then $\left(S,\left.C^{\infty}\left(\mathbb{R}^{2}\right)\right|_{S}\right)$ is a subcartesian differential space but $S$ is not locally closed at $(0,0)$. The vector field $Y=\frac{\partial}{\partial x_{1}}$ on $\mathbb{R}^{2}$ restricts to a derivation $X$ of $C^{\infty}(S)$. For every $x \in \mathbb{R}^{2}$ we have $\mathrm{e}^{t Y} x=\left(x_{1}+t, x_{2}\right)$ for all $t \in \mathbb{R}$. All the maximal integral curves of $X$ have open domains which contain 0 . But $\mathrm{e}^{t X}$ is not a local one-parameter group of local diffeomorphisms of $S$.

\section{Differentiable structure}

For each $n \in \mathbb{Z}_{\geq 0}$ let $\Re_{n}$ be the set of all subsets of which are open in $\mathbb{R}^{n}$. Let $\mathfrak{R}=\bigcup_{n \in \mathbb{Z}_{>0}} \mathfrak{R}_{n}$. Let $S$ be a topological space with $\mathcal{F}$ a subset of $C^{0}(S)$, the set of continuous functions on $S$. For every $U \in \mathfrak{R}$ let $C^{0}(U, S)$ be the set of continuous mappings of $U$ into $S$. Let

$$
\mathcal{F}(U)=\left\{g \in C^{0}(U, S) \mid g^{*} f=f \circ g \in C^{\infty}(U) \text { for every } f \in \mathcal{F}\right\} .
$$

We say that $\mathcal{F}$ is a differentiable structure on $S$ if, given $f \in C^{0}(S)$ such that $g^{*} f \in C^{\infty}(U)$ for every $U \in \Re$ and every $g \in \mathcal{F}(U)$, then $f \in \mathcal{F}$. A differentiable space is a pair $\mathbf{S}=(S, \mathcal{F})$, where $\mathcal{F}=\mathcal{F}_{\mathbf{S}}$ is a differentiable structure on $S$. 
Proposition 4.1 Given a topological space $S$ and a subset $\mathcal{F}$ of $C^{0}(S)$, there is a unique differentiable structure $\mathcal{F}^{*}$ on $S$ such that $\mathcal{F}^{*}(U)=\mathcal{F}(U)$ for every $U \in \mathfrak{R}$.

Proof. Let

$$
\mathcal{F}^{*}=\left\{f \in C^{0}(U) \mid f \circ g \in C^{\infty}(U) \text { for every } U \in \mathfrak{R} \text { and every } g \in \mathcal{F}(U)\right\} .
$$

If $f \in \mathcal{F}$, then for every $U \in \mathfrak{R}$ and every $g \in \mathcal{F}(U)$ we have $g^{*} f \in C^{\infty}(U)$. So $f \in \mathcal{F}^{*}$. Thus $\mathcal{F} \subseteq \mathcal{F}^{*}$. Suppose that $U \in \mathfrak{R}$ and $g \in \mathcal{F}(U)$. Then $g^{*} f \in C^{\infty}(U)$ for every $f \in \mathcal{F}$. Hence $g \in \mathcal{F}^{*}(U)$. So $\mathcal{F}(U) \subseteq \mathcal{F}^{*}(U)$. Let $g \in \mathcal{F}^{*}(U)$. Then $f \circ g \in C^{\infty}(U)$ for every $f \in \mathcal{F}^{*}$. Since $\mathcal{F} \subseteq \mathcal{F}^{*}$, it follows that $f \circ g \in C^{\infty}(U)$ for every $f \in \mathcal{F}$. Hence $g \in \mathcal{F}(U)$. So $\overline{\mathcal{F}}^{*}(U) \subseteq \mathcal{F}(U)$. Consequently, $\mathcal{F}^{*}(U)=\mathcal{F}(U)$ for every $U \in \mathfrak{R}$.

$\mathcal{F}^{*}$ is the differentiable structure on $S$ generated by $\mathcal{F}$. Clearly $\mathcal{F}^{*}$ is a subring of $C^{0}(S)$ with unit, the constant function $\mathbf{1}: S \rightarrow \mathbb{R}: x \mapsto 1$.

Proposition 4.2 Let $M$ be a smooth manifold. Then $C^{\infty}(M)$ is a differentiable structure on $M$.

Proof. Let $m \in M$ and let $V$ be an open subset of $M$ containing $m$ such that $\varphi: V \subseteq M \rightarrow \varphi(V) \subseteq \mathbb{R}^{n}$ is a diffeomorphism. Suppose that $U \in \mathfrak{R}$ and $g: U \rightarrow V \subseteq M$ is a continuous mapping such that $m=g(u)$ for some $u \in U$. For some open subset $W$ of $m$ in $V$ we have $U^{\prime}=g^{-1}(W)$ is an open subset of $U$ containing the point $u$.

Let $\mathcal{F}=C^{\infty}(M)$. We now show that $\mathcal{F}\left(U^{\prime}\right)=C^{\infty}\left(U^{\prime}, U\right)$. Suppose that $g$ : $U^{\prime} \rightarrow U \subseteq M$ satisfies the condition $f \circ g \in C^{\infty}\left(U^{\prime}\right)$ for every $f \in C^{\infty}(M)$, that is, $g \in \mathcal{F}\left(U^{\prime}\right)$. If $g \in C^{\infty}\left(U^{\prime}, U\right)$, then $f \circ g \in C^{\infty}\left(U^{\prime}\right)$ for every $f \in C^{\infty}(M)$. So $g \in \mathcal{F}\left(U^{\prime}\right)$. Thus $C^{\infty}\left(U^{\prime}, U\right) \subseteq \mathcal{F}\left(U^{\prime}\right)$. We now show that $\mathcal{F}\left(U^{\prime}\right) \subseteq C^{\infty}\left(U^{\prime}, U\right)$. Suppose that $g \in \mathcal{F}\left(U^{\prime}\right)$. Then

$$
f \circ g=\left(f \circ \varphi^{-1}\right) \circ(\varphi \circ g)=(\varphi \circ g)^{*}\left(f \circ \varphi^{-1}\right) .
$$

Since $f \in C^{\infty}(M)=\mathcal{F}$, it follows that the function $f \circ \varphi^{-1}: \varphi(V) \subseteq \mathbb{R}^{n} \rightarrow$ $\mathbb{R}$ lies in $C^{\infty}(\varphi(V))$. So $(\varphi \circ g)^{*}\left(C^{\infty}(\varphi(V))\right) \subseteq C^{\infty}\left(U^{\prime}\right)$. Thus the mapping $\varphi \circ g: U^{\prime} \rightarrow \varphi(V) \subseteq \mathbb{R}^{n}$ is smooth. Hence the mapping $g=\varphi^{-1} \circ(\varphi \circ g)$ : $U^{\prime} \rightarrow U \subseteq M$ is smooth. This shows that $\mathcal{F}\left(U^{\prime}\right) \subseteq C^{\infty}\left(U^{\prime}, U\right)$ as desired. So $\mathcal{F}\left(U^{\prime}\right)=C^{\infty}\left(U^{\prime}, U\right)$.

Suppose that $f \in C^{0}(M)$ and $f \circ g \in C^{\infty}\left(U^{\prime}\right)$ for every $U^{\prime} \in \mathfrak{R}$ and every $g \in \mathcal{F}\left(U^{\prime}\right)$. Suppose that $f \notin C^{\infty}(M)$. Then for some $m \in M$ there is an open neighborhood $U$ of $m$ in $M$ such that $(U, \varphi)$ is a chart with $\varphi: U \subseteq M \rightarrow$ $\varphi(U) \subseteq \mathbb{R}^{n}$ a diffeomorphism and the function $f \circ \varphi^{-1}: \varphi(U) \subseteq \mathbb{R}^{n} \rightarrow \mathbb{R}$ is not smooth at $\varphi(m)$. Set $U^{\prime}=\varphi(U)$ and let $g=\varphi^{-1}: U^{\prime}=\varphi(U) \subseteq \mathbb{R}^{n} \rightarrow U \subseteq M$. Then $g \in C^{\infty}\left(U^{\prime}, U\right)=\mathcal{F}\left(U^{\prime}\right)$ but $g^{*} f: U^{\prime} \rightarrow \mathbb{R}$ is not smooth at $\varphi(m) \in U^{\prime}$. This contradicts our hypothesis that $g \in \mathcal{F}\left(U^{\prime}\right)$. Hence $f \in C^{\infty}(M)$. In other words, $C^{\infty}(M)$ is a differentiable structure on $M$. 
Let $S$ be a differential space. Define

$$
\Gamma_{0} C^{\infty}(S)=\left\{c \in C^{0}(\mathbb{R}, S) \mid f_{\circ} c \in C^{\infty}(\mathbb{R}) \text { for all } f \in C^{\infty}(S)\right\} .
$$

From the definition of the differential space topology on $S$, it follows that $\Gamma_{0} C^{\infty}(S)=C^{0}(\mathbb{R}, S)$. Also define

$$
\Phi_{0} \Gamma_{0} C^{\infty}(S)=\left\{f \in C^{0}(S) \mid f_{\circ} c \in C^{\infty}(\mathbb{R}) \text { for all } c \in \Gamma_{0} C^{\infty}(S)\right\} .
$$

Clearly $C^{\infty}(S) \subseteq \Phi_{0} \Gamma_{0} C^{\infty}(S)$. We say that $C^{\infty}(S)$ is continuously reflexive if $\Phi_{0} \Gamma_{0} C^{\infty}(S) \subseteq C^{\infty}(S)$. We have

Theorem 4.3 If $C^{\infty}(S)$ is a continuously reflexive differential structure on the differential space $\left(S, C^{\infty}(S)\right)$, then $C^{\infty}(S)$ is a differentable structure on $S$ in the sense of Smith.

Proof. Let $S$ be a topological space with $\mathcal{F}$ a subset of $C^{0}(S)$, the set of continuous functions on $S$. For every $U \in \mathfrak{R}$ let $C^{0}(U, S)$ be the set of continuous mappings of $U$ into $S$. Set

$$
\mathcal{F}(U)=\left\{g \in C^{0}(U, S) \mid g^{*} f=f \circ g \in C^{\infty}(U) \text { for every } f \in \mathcal{F}\right\} .
$$

Recall that $\mathcal{F}$ is a differentiable structure on $S$ if $f \in C^{0}(S)$ lies in $\mathcal{F}$ whenever the condition

$$
g^{*} f \in C^{\infty}(U) \text { for every } U \in \mathfrak{R} \text { and every } g \in \mathcal{F}(U)
$$

is satisfied. Take $\mathcal{F}=C^{\infty}(S)$. Then definition (6) reads: for every $U \in \mathfrak{R}$

$$
C^{\infty}(S)(U)=\left\{g \in C^{0}(U, S) \mid g^{*} f \in C^{\infty}(U) \text { for every } f \in C^{\infty}(S)\right\} .
$$

So condition (7) that $C^{\infty}(S)$ is a differentiable structure on $S$ reads: a continuous function $f$ on $S$ lies in $C^{\infty}(S)$ whenever the condition

$$
g^{*} f \in C^{\infty}(U) \text { for every } U \in \mathfrak{R} \text { and every } g \in C^{\infty}(S)(U)
$$

is satisfied. Let $f \in C^{0}(S)$ and suppose that condition (9) and definition (8) hold. Specializing to the case when $U=\mathbb{R} \in \mathfrak{R}_{1}$ definition (8) reads:

$C^{\infty}(S)(\mathbb{R})=\left\{g \in C^{0}(\mathbb{R}, S) \mid g^{*} f \in C^{\infty}(\mathbb{R})\right.$ for every $\left.f \in C^{\infty}(S)\right\}=\Gamma_{0} C^{\infty}(S)$, using (44), and condition (9) reads:

$$
g^{*} f \in C^{\infty}(\mathbb{R}) \text { for every } g \in \Gamma_{0} C^{\infty}(S) .
$$

From equation (5) it follows that $f \in \Phi_{0}\left(\Gamma_{0}\left(C^{\infty}(S)\right)\right)$. By hypothesis $C^{\infty}(S)$ is continuously reflexive, that is, $\Phi_{0}\left(\Gamma_{0}\left(C^{\infty}(S)\right)\right)=C^{\infty}(S)$. So $f \in C^{\infty}(S)$. Thus $C^{\infty}(S)$ is a differentiable structure on $S$.

Let $\mathbf{S}=\left(S, \mathcal{F}_{\mathbf{S}}\right)$ and $\mathbf{S}^{\prime}=\left(S^{\prime}, \mathcal{F}_{\mathbf{S}^{\prime}}\right)$ be differentiable spaces and let $h: \mathbf{S} \rightarrow \mathbf{S}^{\prime}$ be a continuous map. $h$ is a differentiable mapping if and only if $h^{*}\left(\mathcal{F}_{\mathbf{S}^{\prime}}\right) \subseteq \mathcal{F}_{\mathbf{S}}$. 
Proposition 4.4 A continuous function $f: S \rightarrow \mathbb{R}$ is a differentiable map $\mathbf{f}: \mathbf{S} \rightarrow \mathbf{R}=\left(\mathbb{R}, C^{\infty}(\mathbb{R})\right)$ if and only if $f \in \mathcal{F}_{\mathbf{S}}$.

Proof: This follows immediately from the definition of smooth map between differentiable spaces.

Proposition 4.5 A map $\varphi:\left(S_{1}, C^{\infty}\left(S_{1}\right)\right) \rightarrow\left(S_{1}, C^{\infty}\left(S_{1}\right)\right)$ of differential spaces with continuously reflexive differential structures is smooth if and only if it is a differentable mapping between the differentiable spaces $\mathbf{S}_{1}=\left(S_{1}, C^{\infty}\left(S_{1}\right)\right)$ and $\mathbf{S}_{2}=\left(S_{2}, C^{\infty}\left(S_{2}\right)\right)$.

Proof. The map $\varphi$ between the differential spaces $S_{1}$ and $S_{2}$ is smooth if and only if $\varphi^{*}\left(C^{\infty}\left(S_{2}\right)\right) \subseteq C^{\infty}\left(S_{1}\right)$ if and only if it is a differentable map between differentiable spaces $\mathbf{S}_{1}$ and $\mathbf{S}_{2}$.

We now give some results on differentable structures that we will need when discussing differential forms on differentiable spaces.

Proposition 4.6 If $g_{1}, \ldots, g_{n} \in \mathcal{F}_{\mathbf{S}}, \phi \in C^{\infty}\left(\mathbb{R}^{n}\right)$, and $U \in \mathfrak{R}$, then lies in $\mathcal{F}_{\mathbf{S}}$.

$$
f=\phi\left(g_{1}, \ldots, g_{n}\right): U \rightarrow \mathbb{R}: x \mapsto f(x)=\phi\left(g_{1}(x), \ldots, g_{n}(x)\right)
$$

Proof. For every $U \in \mathfrak{R}$ and every $h: U \rightarrow S$ in $\mathcal{F}_{\mathbf{S}}(U)$ we have $g_{i} \circ h \in C^{\infty}(U)$. Hence $f \circ h \in C^{\infty}(U)$, which implies that $f \in \mathcal{F}_{\mathbf{S}}$.

Let $\mathbf{S}^{\prime}=\left(S^{\prime}, \mathcal{F}_{\mathbf{S}^{\prime}}\right)$ and $\left(\mathbf{S}^{\prime \prime}, \mathcal{F}_{\mathbf{S}^{\prime \prime}}\right)$ be differentiable spaces. Define the product $\mathbf{S}^{\prime} \times \mathbf{S}^{\prime \prime}=\left(S^{\prime} \times S^{\prime \prime}, \mathcal{F}_{\mathbf{S}^{\prime} \times \mathbf{S}^{\prime \prime}}\right)$, where $\mathcal{F}_{\mathbf{S}^{\prime} \times \mathbf{S}^{\prime \prime}}$ is the set of $f \in C^{0}\left(S^{\prime} \times S^{\prime \prime}\right)$ such that $f\left(g^{\prime}, g^{\prime \prime}\right) \in C^{\infty}(U)$ for every $U \in \mathfrak{R}$ and every $g^{\prime} \in \mathcal{F}_{\mathbf{S}^{\prime}}$ and $g^{\prime \prime} \in \mathcal{F}_{\mathbf{S}^{\prime \prime}}$.

Lemma 4.7 $\mathcal{F}_{\mathbf{S}^{\prime} \times \mathbf{S}^{\prime \prime}}$ is a differentiable structure.

Proof. Let $f: S \rightarrow \mathbb{R}$ be a continuous function such that $f \circ h \in C^{\infty}(U)$ for every $U \in \mathfrak{R}$ and every $h \in \mathcal{F}_{\mathbf{S}^{\prime} \times \mathbf{S}^{\prime \prime}}$. Consider $h=\left(h^{\prime}, h^{\prime \prime}\right)$ with $h^{\prime} \in \mathcal{F}_{\mathbf{S}^{\prime}}(U)$ and $h^{\prime \prime} \in \mathcal{F}_{\mathbf{S}^{\prime \prime}}(U)$. For every $g \in \mathcal{F}_{\mathbf{S}^{\prime} \times \mathbf{S}^{\prime \prime}}$ we have $g \circ h \in C^{\infty}(U)$. This implies that $h \in \mathcal{F}_{\mathbf{S}^{\prime} \times \mathbf{S}^{\prime \prime}}(U)$. Thus $f \circ h \in C^{\infty}(U)$, that is, $f \in \mathcal{F}_{\mathbf{S}^{\prime} \times \mathbf{S}^{\prime \prime}}$.

Hence the product $\mathbf{S}^{\prime} \times \mathbf{S}^{\prime \prime}=\left(S^{\prime} \times S^{\prime \prime}, \mathcal{F}_{\mathbf{S}^{\prime} \times \mathbf{S}^{\prime \prime}}\right)$ is a differentiable space.

Suppose that $g_{1}^{\prime}, \ldots, g_{n}^{\prime} \in \mathcal{F}_{\mathbf{S}^{\prime}}$ and $g_{1}^{\prime \prime}, \ldots, g_{m}^{\prime \prime} \in \mathcal{F}_{\mathbf{S}^{\prime \prime}}$ are given and that $\phi \in C^{\infty}\left(\mathbb{R}^{n+m}\right)$. Let $\pi^{\prime}: S^{\prime} \times S^{\prime \prime} \rightarrow S^{\prime}$ and $\pi^{\prime \prime}: S^{\prime} \times S^{\prime \prime} \rightarrow S^{\prime \prime}$ be the natural projections. Let $f_{i}^{\prime}=g_{i}^{\prime} \circ \pi^{\prime}$ and $f_{j}^{\prime \prime}=g_{j}^{\prime \prime} \circ \pi^{\prime \prime}$. Then

$$
f=\phi\left(f_{1}^{\prime}, \ldots, f_{n}^{\prime}, f_{1}^{\prime \prime}, \ldots, f_{m}^{\prime \prime}\right)
$$

lies in $\mathcal{F}_{\mathbf{S}^{\prime} \times \mathbf{S}^{\prime \prime}}$. Let $\overline{\mathcal{F}}_{\mathbf{S}^{\prime} \times \mathbf{S}^{\prime \prime}}$ be the subset of all functions in $\mathcal{F}_{\mathbf{S}^{\prime} \times \mathbf{S}^{\prime \prime}}$ which can be written in the form (11). Then $\overline{\mathcal{F}}_{\mathbf{S}^{\prime} \times \mathbf{S}^{\prime \prime}}$ is a linear subspace of the real vector space $\mathcal{F}_{\mathbf{S}^{\prime} \times \mathbf{S}^{\prime \prime}}$.

A differentiable map $h: \mathbf{S}^{\prime} \times \mathbf{S}^{\prime \prime} \rightarrow \mathbf{T}$ is well behaved if $g \circ h \in \overline{\mathcal{F}}_{\mathbf{S}^{\prime} \times \mathbf{S}^{\prime \prime}}$ for every $g \in \mathcal{F}_{\mathbf{T}}$. Clearly the natual projections $\pi^{\prime}$ and $\pi^{\prime \prime}$ are well behaved differentiable mappings. We will need this notion in section 5 , when proving the Poincaré lemma. 
The collection of differentiable spaces and differentiable maps form a category $\mathcal{D}$ under composition. The category of smooth manifolds and smooth maps is a full subcategory of $\mathcal{D}$. Let $\mathcal{K}$ be the category of rings with unit and ring homomorphisms. We have a contravariant functor $F: \mathcal{D} \rightarrow \mathcal{K}$, which sends a differentiable space $\mathbf{S}$ to its differentiable structure $\mathcal{F}_{\mathbf{S}}$, which is a ring with unit and sends a differentiable map between differentiable spaces to a ring homomorphism between the differential structures $\mathcal{F}_{\mathbf{S}^{\prime}}$ and $\mathcal{F}_{\mathbf{S}}$. More precisely, if $h: \mathbf{S}=\left(S, \mathcal{F}_{\mathbf{S}}\right) \rightarrow \mathbf{S}^{\prime}=\left(S^{\prime}, \mathcal{F}_{\mathbf{S}^{\prime}}\right)$ is a differentiable map, then $h^{*}: \mathcal{F}_{\mathbf{S}^{\prime}} \rightarrow \mathcal{F}_{\mathbf{S}}: f \mapsto f \circ h$ is a ring homomorphism. Let $U$ be an open subset of $S$ and let $\mathcal{F}_{U}$ be the differentiable structure on $U$ generated by $\left.f\right|_{U}$ where $f \in \mathcal{F}_{\mathbf{S}}$. Let $\left.\mathbf{S}\right|_{U}=\left(U, \mathcal{F}_{U}\right)$ and let $\left.\iota_{\mathbf{S}}\right|_{U}:\left.\mathbf{S}\right|_{U} \rightarrow \mathbf{S}$ be the inclusion map. This defines a local category $\mathcal{D}$. Similarly, we can define a local category $\mathcal{S}^{0}$ with objects consisting of pairs of an open subset $U$ of the topological space $S$ and the set of continuous functions on $S$ restricted to $U$. Thus the objects of $\mathcal{S}^{0}$ are $S_{U}=\left(U, C^{0}(U)\right)$. The maps in $\mathcal{S}^{0}$ are the inclusions $\iota_{U}: U \mapsto S$. For every object $\mathbf{S}$ in the local category $\mathcal{D}$ we have a covariant functor $T_{\mathbf{S}}: \mathcal{S}^{0} \rightarrow \mathcal{D}$ whose value at $\mathbf{S}_{U}$ is the open subset $U$ of $S$ and whose value at the map $\left.\iota_{U}\right|_{V}$ is the map $\left.\left(\left.\iota_{\mathbf{S}}\right|_{U}\right)\right|_{V}$ for every open subset $U$ and $V$ of $S$ such that $V \subseteq U$. For every $\mathbf{S} \in \mathcal{D}$ the map

$$
F^{0}=F_{\circ} T_{\mathbf{S}}: \mathcal{S}^{0} \rightarrow \mathcal{K}: U \subseteq S \mapsto \mathcal{F}_{\left.\mathbf{S}\right|_{U}}
$$

is a sheaf on $S$ with values in the category $\mathcal{K}$.

\section{Exterior algebra on a differentiable space}

In this section we construct an exterior algebra of differential forms on a differentiable space and prove a version of de Rham's theorem. We follow the arguments of Smith [13] quite closely and give the details.

\subsection{Differential forms}

In this subsection we construct the graded exterior algebra of differential forms and exterior derivative on the differentiable space $S$.

A $p$-dimensional cube $J^{p} \subseteq \mathbb{R}^{p}$ with $p>0$ is a closed subset of $\mathbb{R}^{p}$ bounded by $2 p$ axis parallel hyperplanes. A map $\sigma: J^{p} \subseteq \mathbb{R}^{p} \rightarrow S$ is a singular $p$-cube in the differentiable space $\mathbf{S}=\left(S, \mathcal{F}_{\mathbf{S}}\right)$ if there is a $U \in \mathfrak{R}_{p}$ with $J^{p} \subseteq U \subseteq \mathbb{R}^{p}$ and a map $f: U \rightarrow S$ in $\mathcal{F}_{\mathbf{S}}(U)$ such that $\sigma=\left.f\right|_{J^{p}}$. We say that the singular $p$-cube $\sigma$ is represented by the mapping $f \in \mathcal{F}_{\mathbf{S}}(U)$. Let $K_{p}(\mathbf{S})$ be the set of all singular $p$-cubes in $\mathbf{S}$. Let $C^{p}(\mathbf{S})$ be the set of all real valued functions $\alpha: K_{p}(\mathbf{S}) \rightarrow \mathbb{R}$. An element of $C^{p}(\mathbf{S})$ is a singular p-cochain on $\mathbf{S}$. Clearly, $C^{p}(\mathbf{S})$ is a real vector space. When $p=0$ let $J^{0}=\{0\}$ in $\mathbb{R}^{0}=\{0\}$. A singular 0 -cube is a mapping $\sigma: J^{0} \subseteq \mathbb{R}^{0} \rightarrow S$ which sends 0 to a point $x \in S$. Hence $K_{0}(\mathbf{S})$, the set of singular 0-cubes in $\mathbf{S}$, is $S$. The set $C^{0}(\mathbf{S})$ of singular 0-cochains on $\mathbf{S}$ is $\mathcal{F}_{\mathbf{S}}$, the differentiable structure of $\mathbf{S}$. 
For every $p>0$ define the mapping

$$
\lambda_{p}: \mathcal{F}_{\mathbf{S}}^{p+1}=\overbrace{\mathcal{F}_{\mathbf{S}} \times \cdots \times \mathcal{F}_{\mathbf{S}}}^{p+1} \rightarrow C^{p}(\mathbf{S}):\left(f_{0}, \ldots, f_{p}\right) \mapsto \lambda_{p}\left(f_{0}, \ldots, f_{p}\right),
$$

where $f_{i} \in \mathcal{F}_{\mathbf{S}}$. To specify $\lambda_{p}\left(f_{0}, \ldots, f_{p}\right)$, we need to give its value on a singular $p$-cube $\sigma: J^{p} \rightarrow S$ represented by $f \in \mathcal{F}_{\mathbf{S}}(U)$. Define

$$
\lambda_{p}\left(f_{0}, \ldots, f_{p}\right)(\sigma)=\int_{J^{p}} g_{0} \operatorname{det} D G\left(t_{1}, \ldots, t_{p}\right) \mathrm{d} t_{1} \cdots \mathrm{d} t_{p},
$$

where

$$
\begin{aligned}
G: U & \subseteq \mathbb{R}^{p} \rightarrow \mathbb{R}^{p}: \\
\mathbf{t} & =\left(t_{1}, \ldots, t_{p}\right) \mapsto\left(g_{1}(\mathbf{t}), \ldots, g_{p}(\mathbf{t})\right)=\left(\left(f_{1} \circ f\right)(\mathbf{t}), \ldots,\left(f_{p} \circ f\right)(\mathbf{t})\right)
\end{aligned}
$$

is a smooth mapping and $g_{0}=f_{0} \circ$. The Jacobian $\operatorname{det} D G$ of the mapping $G$ will be denoted by $\frac{\partial\left(g_{1}, \ldots, g_{p}\right)}{\partial\left(t_{1}, \ldots, t_{p}\right)}$. Since the right hand side of (13) depends only on the singular $p$-cube $\sigma$ and not on its representation $f$, it follows that $\lambda_{p}\left(f_{0}, \ldots, f_{p}\right)$ is a singular $p$-cochain. Hence the mapping $\lambda_{p}(12)$ is well defined. When $p=0$ the set $C^{0}(\mathbf{S})$ of singular 0 -cochains on $\mathbf{S}$ is $\mathcal{F}_{\mathbf{S}}$. The map $\lambda_{0}$ : $\mathcal{F}_{\mathbf{S}} \rightarrow C^{0}(\mathbf{S})=\mathcal{F}_{\mathbf{S}}$ is the identity map, because $\lambda_{0}\left(f_{0}\right) \sigma=f_{0} \circ$, where $\sigma$ is a singular 0 -cube.

For $p>0$ let $G^{p}(\mathbf{S})$ be the real linear vector subspace of $C^{p}(\mathbf{S})$ spanned by the image of the map $\lambda_{p}$. When $p=0$ set $G^{0}(\mathbf{S})=\mathcal{F}_{\mathbf{S}}$. We call $G^{p}(\mathbf{S})$ the real vector space of differential $p$ forms on the differentiable space $\mathbf{S}$.

Let $F: \mathbf{S} \rightarrow \mathbf{S}^{\prime}$ be a differentiable mapping. Then $F$ induces a real linear mapping

$$
F^{*}: C^{p}\left(\mathbf{S}^{\prime}\right) \rightarrow C^{p}(\mathbf{S}): \lambda_{p}\left(f_{1}^{\prime}, \ldots, f_{p}^{\prime}\right) \mapsto \lambda_{p}\left(f_{1}, \ldots, f_{p}\right),
$$

where $f_{i}=f_{i}^{\prime} \circ F$. We now show that the induced map $F^{*}$ on cochains is well defined. Let $\sigma: J^{p} \rightarrow S$ be a singular $p$-cube represented by $f \in \mathcal{F}_{\mathbf{S}}(U)$. Then $F_{\circ} \sigma \in C^{p}\left(\mathbf{S}^{\prime}\right)$. Check: $F_{\circ} \sigma: J^{p} \rightarrow S^{\prime}$ and $F_{\circ} f: U \rightarrow S^{\prime}$ is in $\mathcal{F}_{\mathbf{S}^{\prime}}(U)$ because $g \circ(F \circ f) \in C^{\infty}(U)$ for every $g \in \mathcal{F}_{\mathbf{S}^{\prime}}$. The last assertion follows because $g_{\circ}(F \circ f)=F^{*}(g) \circ f \in C^{\infty}(U)$ for $F^{*}(g) \in \mathcal{F}_{\mathbf{S}}$, since $f \in \mathcal{F}_{\mathbf{S}}(U)$. Now $\left.(F \circ f)\right|_{J^{p}}=F_{\circ}\left(\left.f\right|_{J^{p}}\right)=F_{\circ} \sigma$. Thus $F_{\circ} \sigma \in C^{p}\left(\mathbf{S}^{\prime}\right)$. The following computation finishes the verification of equation (14).

$$
\begin{aligned}
\lambda_{p}\left(f_{1}^{\prime}, \ldots, f_{p}^{\prime}\right)(F \circ \sigma) & =\int_{J^{p}}\left(f_{0}^{\prime} \circ F\right) \frac{\partial\left(f_{1}^{\prime} \circ(F \circ f), \ldots, f_{p^{\circ}}^{\prime}(F \circ f)\right)}{\partial\left(t_{1}, \ldots, t_{p}\right)} \mathrm{d} t_{1} \cdots \mathrm{d} t_{p} \\
& =\int_{J^{p}} f_{0} \frac{\partial\left(f_{1} \circ f, \ldots, f_{p^{\circ}} f\right)}{\partial\left(t_{1}, \ldots, t_{p}\right)} \mathrm{d} t_{1} \cdots \mathrm{d} t_{p} \\
& =\lambda_{p}\left(f_{0}, \ldots, f_{p}\right)(\sigma) .
\end{aligned}
$$


Example 5.1.1 Let $U \in \mathfrak{R}$. Then $U$ is a smooth manifold. So $\mathbf{U}=\left(U, C^{\infty}(U)\right)$ is a differentiable space with differential structure $C^{\infty}(U)$. Let $F^{p}(\mathbf{U})$ be the real vector space of differentiable $p$-forms on $U$. The map

$$
\Phi: G^{p}(\mathbf{U}) \rightarrow F^{p}(\mathbf{U}): \lambda_{p}\left(g_{0}, \ldots, g_{p}\right) \mapsto g_{0} \mathrm{~d} g_{1} \wedge \cdots \wedge \mathrm{d} g_{p},
$$

where $g_{i} \in C^{\infty}(U)$, is an isomorphism. The mapping $\Phi$ is well defined. For every singular $p$-cube $\sigma: J^{p} \subseteq U \rightarrow U$ in $U$ we have

$$
\int_{J^{p}} g_{0} \mathrm{~d} g_{1} \wedge \cdots \wedge \mathrm{d} g_{p}=\lambda_{p}\left(g_{0}, \ldots, g_{p}\right) \sigma .
$$

Check: The singular $p$-cube $\sigma$ is represented by id : $U \rightarrow U$. By definition

$$
\begin{aligned}
\lambda_{p}\left(g_{0}, \ldots, g_{p}\right) \sigma & =\int_{J^{p}}\left(g_{0} \circ \mathrm{id}\right) \frac{\partial\left(g_{1} \circ \mathrm{id}, \ldots, g_{p^{\circ}} \mathrm{id}\right)}{\partial\left(t_{1}, \ldots, t_{p}\right)} \mathrm{d} t_{1} \cdots \mathrm{d} t_{p} \\
& =\int_{J^{p}} g_{0} \frac{\partial\left(g_{1}, \ldots, g_{p}\right)}{\partial\left(t_{1}, \ldots, t_{p}\right)} \mathrm{d} t_{1} \cdots \mathrm{d} t_{p} \\
& =\int_{J^{p}} g_{0} \mathrm{~d} g_{1} \wedge \cdots \wedge \mathrm{d} g_{p} .
\end{aligned}
$$

Clearly the map $\Phi$ (15) is a real linear mapping. It is injective, for if $\lambda_{p}\left(g_{0}, \ldots, g_{p}\right)$ $=0$, then $\lambda_{p}\left(g_{0}, \ldots g_{p}\right) \sigma=0$ for all singular $p$-cubes $\sigma$. From (16) we obtain $\int_{J^{p}} g_{0} \mathrm{~d} g_{1} \wedge \cdots \wedge \mathrm{d} g_{p}=0$ for every $p$-cube $J^{p}$ in $\mathbb{R}^{p}$. Thus $g_{0} \mathrm{~d} g_{1} \wedge \cdots \wedge \mathrm{d} g_{p}=0$. Equation (16) shows that the mapping $\Phi$ is surjective. Consequently, $\Phi$ is an isomorphism.

If $\alpha=\lambda_{p}\left(g_{0}, \ldots, g_{p}\right) \in G^{p}(\mathbf{S})$ with $g_{i} \in \mathcal{F}_{\mathbf{S}}$ and if $f \in \mathcal{F}_{\mathbf{S}}(U)$ represents the singular $p$-cube $\sigma: J^{p} \rightarrow S$, then $f^{*}(\alpha)=\left(g_{0} \circ f\right) \mathrm{d}\left(g_{1} \circ f\right) \wedge \cdots \wedge \mathrm{d}\left(g_{p} \circ f\right) \in$ $F^{p}(\mathbf{U})$, where $g_{i} \circ f \in C^{\infty}(U)$.

\subsection{Exterior derivative}

First we prove the existence of an exterior product on the space of differential forms on the differentiable space $\mathbf{S}$.

Proposition 5.2.1 There is a unique bilinear map

$$
\begin{aligned}
& \Lambda: G^{p}(\mathbf{S}) \times G^{q}(\mathbf{S}) \rightarrow G^{p+q}(\mathbf{S}): \\
& \quad\left(\lambda_{p}\left(f_{0}, \ldots, f_{p}\right), \lambda_{q}\left(\bar{f}_{0}, \ldots, \bar{f}_{q}\right)\right) \mapsto \lambda_{p+q}\left(f_{0}, \bar{f}_{0}, f_{1}, \ldots, f_{p}, \bar{f}_{1}, \ldots, \bar{f}_{q}\right),
\end{aligned}
$$

where $f_{i}, \bar{f}_{j} \in G^{0}(\mathbf{S})=\mathcal{F}_{\mathbf{S}}$.

Proof. Let $\alpha=\lambda_{p}\left(f_{0}, \ldots, f_{p}\right)$ and $\beta=\lambda_{q}\left(\bar{f}_{0}, \ldots, \bar{f}_{q}\right)$. Let $\sigma: J^{p+q} \rightarrow S$ be a singular $p+q$ cube represented by the map $f \in \mathcal{F}_{\mathbf{S}}(U)$. Then $f^{*}(\alpha) \in F^{p}(U)$ and $f^{*}(\beta) \in F^{q}(U)$. Moreover,

$$
\Lambda(\alpha, \beta) \sigma=\int_{J^{p+q}} f^{*}(\alpha) \wedge f^{*}(\beta) .
$$


We write $\alpha \wedge \beta$ instead of $\Lambda(\alpha, \beta)$.

Next we define the exterior derivative operator $\mathrm{d}$. There is a unique real linear mapping

$$
\mathrm{d}: G^{p}(\mathbf{S}) \rightarrow G^{p+1}(\mathbf{S}): \lambda_{p}\left(f_{0}, \ldots, f_{p}\right) \mapsto \lambda_{p+1}\left(\mathbf{1}, f_{0}, \ldots, f_{p}\right),
$$

where $f_{i} \in G^{0}(\mathbf{X})$.

Proposition 5.2.2 The map $\mathrm{d}(17)$ is the coboundary operator with $\mathrm{d}^{2}=0$.

Proof. For $f_{i} \in G^{0}(\mathbf{S})=\mathcal{F}_{\mathbf{S}}$ we have

$$
\mathrm{d}^{2} \lambda_{p}\left(f_{0}, \ldots, f_{p}\right)=\mathrm{d} \lambda_{p+1}\left(\mathbf{1}, f_{0}, \ldots, f_{p}\right)=\lambda_{p+2}\left(\mathbf{1}, \mathbf{1}, f_{0}, \ldots, f_{p}\right) .
$$

From equation (13) we get

$$
\lambda_{p+2}\left(\mathbf{1}, \mathbf{1}, f_{0}, \ldots, f_{p}\right)=\int_{J^{p+2}} \mathbf{1} \frac{\partial\left(\mathbf{1}, g_{0}, \ldots, g_{p}\right)}{\partial\left(t_{1}, \ldots, t_{p+1}\right)} \mathrm{d} t_{1} \cdots \mathrm{d} t_{p+1},
$$

where $g_{i}=f_{i}$ 。 $f$ and $f \in \mathcal{F}_{\mathbf{S}}$ represents the singular $p+1$-cube $\sigma: J^{p+1} \rightarrow S$. Since $\frac{\mathrm{d} \mathbf{1}}{\mathrm{d} t_{i}}=0$, the Jacobian $\frac{\partial\left(\mathbf{1}, g_{0}, \ldots, g_{p}\right)}{\partial\left(t_{1}, \ldots, t_{p+1}\right)}=0$. So $\lambda_{p+2}\left(\mathbf{1}, \mathbf{1}, f_{0}, \ldots, f_{p}\right)=0$. Consequently, $\mathrm{d}^{2}=0$ on $G^{p}(\mathbf{S})$ for every $p \geq 0$.

From the definition of exterior product and exterior derivative we get

$$
\lambda_{p}\left(f_{0}, \ldots, f_{p}\right)=\lambda_{0}\left(f_{0}\right) \wedge \lambda_{1}\left(\mathbf{1}, f_{1}\right) \wedge \cdots \lambda_{1}\left(\mathbf{1}, f_{p}\right)=f_{0} \mathrm{~d} f_{1} \wedge \cdots \wedge \mathrm{d} f_{p},
$$

since $\lambda_{0}\left(f_{0}\right)=f_{0}$. It is straightforward to verify that

$$
\mathrm{d}(\alpha \wedge \beta)=\mathrm{d} \alpha \wedge \beta+(-1)^{\operatorname{deg} \alpha} \alpha \wedge \mathrm{d} \beta .
$$

Thus $\left(G(\mathbf{S})=\sum_{p \geq 0} \oplus G^{p}(\mathbf{S}), \mathrm{d}\right)$ is a (graded) differential exterior algebra. If $F: \mathbf{S} \rightarrow \mathbf{S}^{\prime}$ is a differentiable map in the local category $\mathcal{D}$ of differential spaces and maps, then

$$
F^{*}: G\left(\mathbf{S}^{\prime}\right) \rightarrow G(\mathbf{S}): \alpha \mapsto F^{*}(\alpha)
$$

is a homomorphism of (graded) differential exterior algebras. Let $\mathcal{A}$ be the category of graded differential algebras and homomorphisms. Then the mapping $G: \mathcal{D} \rightarrow \mathcal{A}$, which sends the differentiable space $\mathbf{S}$ to the exterior algebra $G(\mathbf{S})$ and the mapping $F: \mathbf{S} \rightarrow \mathbf{S}^{\prime}$ to the homomorphism $F^{*}: G\left(\mathbf{S}^{\prime}\right) \rightarrow G(\mathbf{S})$ is a contravariant functor.

When $\mathbf{S}=\mathbf{S}^{\prime} \times \mathbf{S}^{\prime \prime}$ the space $\overline{\mathcal{F}}_{\mathbf{S}^{\prime} \times \mathbf{S}^{\prime \prime}}$, see page 12, generates an exterior differential subalgebra $\bar{G}\left(\mathbf{S} \times \mathbf{S}^{\prime \prime}\right)$ of $G\left(\mathbf{S}^{\prime} \times \mathbf{S}^{\prime \prime}\right)$. If the differentiable map $h$ : $\mathbf{S}^{\prime} \times \mathbf{S}^{\prime \prime} \rightarrow \mathbf{T}$ is well behaved, then it induces a real linear mapping $h^{*}: G(\mathbf{T}) \rightarrow$ $\bar{G}\left(\mathbf{S}^{\prime} \times \mathbf{S}^{\prime \prime}\right)$.

Finally we prove the chain rule. For $i=1, \ldots, n$ let $g_{i} \in \mathcal{F}_{\mathbf{S}}$ and let $F \in$ $C^{\infty}\left(\mathbb{R}^{n}\right)$. For $\phi=F\left(g_{1}, \ldots, g_{n}\right) \in \mathcal{F}_{\mathbf{S}}$ we have

$$
\mathrm{d} \phi=\sum_{i=1}^{n} \frac{\partial F}{\partial g_{i}} \mathrm{~d} g_{i} .
$$


Proof. Let $\sigma: J^{1} \rightarrow S$ be a singular 1-cube represented by $f \in \mathcal{F}_{\mathbf{S}}(U)$. Then

$$
\begin{aligned}
\mathrm{d} \phi(\sigma) & =\lambda_{1}(\mathbf{1}, \phi) \sigma=\int_{J^{1}} \frac{\mathrm{d}(\phi \circ f)}{\mathrm{d} t} \mathrm{~d} t \\
& =\int_{J^{1}} \frac{\mathrm{d}}{\mathrm{d} t} F\left(g_{1} \circ f, \ldots, g_{n^{\circ}} f\right) \mathrm{d} t=\int_{J^{1}} \sum_{i=1}^{n} \frac{\partial F}{\partial g_{i}} \frac{\mathrm{d}\left(g_{i} \circ f\right)}{\mathrm{d} t} \mathrm{~d} t \\
& =\sum_{i=1}^{n} \frac{\partial F}{\partial g_{i}} \int_{J^{1}} \frac{\mathrm{d}\left(g_{i} \circ f\right)}{\mathrm{d} t} \mathrm{~d} t=\sum_{i=1}^{n} \frac{\partial F}{\partial g_{i}} \lambda_{1}\left(\mathbf{1}, g_{i}\right) \sigma \\
& =\left(\sum_{i=1}^{n} \frac{\partial F}{\partial g_{i}} \mathrm{~d} g_{i}\right) \sigma .
\end{aligned}
$$

\subsection{The Poincaré lemma}

The unit interval $I=[0,1] \subseteq \mathbb{R}$ with $C^{\infty}(I)=\left.C^{\infty}(\mathbb{R})\right|_{I}$ is a differentiable space $\mathbf{I}=\left(I, C^{\infty}(I)\right)$. A differentiable space $\mathbf{S}=\left(S, \mathcal{F}_{\mathbf{S}}\right)$ is differentiably contractible if there is a well behaved differentiable map $h: \mathbf{I} \times \mathbf{S} \rightarrow \mathbf{S}$ such that $h(1, x)=x$ and $h(0, x)=x_{0}$ for all $x \in S$ with $x_{0}$ a point in $S$. The map $h$ is called a contraction of $S$.

Proposition 5.3.1 Let $\mathbf{S}$ be a differentiably contractible differentiable space and let $\alpha \in G^{p+1}(\mathbf{S})$ such that $\mathrm{d} \alpha=0$. Then $\alpha=\mathrm{d} \beta$ for some $\beta \in G^{p}(\mathbf{S})$.

Proof. For $p \geq 0$ let $K_{p}(\mathbf{S})$, the set of singular $p$-chains of $\mathbf{S}$, be the free abelian group generated by $C_{p}(\mathbf{S})$, the set of singular $p$-cubes of $\mathbf{S}$. Here $K_{0}(\mathbf{S})=S$. Let $\sigma: J^{p+1} \rightarrow S$ be an element of $C_{p+1}(\mathbf{S})$, where

$$
J^{p+1}=\left\{\left(t_{1}^{\prime}, \ldots, t_{p+1}^{\prime}\right) \in \mathbb{R}^{p+1} \mid A_{i}^{-} \leq t_{i}^{\prime} \leq A_{i}^{+}, 1 \leq i \leq p+1\right\}
$$

and $A_{i}^{ \pm} \in \mathbb{R}$. For $1 \leq i \leq p$ let

$$
\begin{aligned}
& J_{i}^{p+1}=\left\{\left(t_{1}, \ldots, t_{p}\right)\right. \in \mathbb{R}^{p} \mid \\
&\left.A_{j}^{-} \leq t_{j} \leq A_{j}^{+}, 1 \leq j<i \& A_{j+1}^{-} \leq t_{j} \leq A_{j+1}^{+} i \leq j \leq p\right\} .
\end{aligned}
$$

Then $J_{i}^{p+1}$ is a $p$-cube. For $1 \leq i \leq p$ let

$$
\phi_{i}^{ \pm}: \mathbb{R}^{p} \rightarrow \mathbb{R}^{p+1}:\left(t_{1}, \ldots, t_{p}\right) \mapsto\left(t_{1}^{\prime}, \ldots, t_{p+1}^{\prime}\right)=\left(t_{1}, \ldots, t_{i-1}, A_{i}^{ \pm}, t_{i}, \ldots, t_{p}\right) .
$$

So

$$
\begin{aligned}
\phi_{i}^{ \pm}\left(J_{i}^{p+1}\right) & =\left\{\left(t_{1}^{\prime}, \ldots, t_{p+1}^{\prime}\right)=\left(t_{1}, \ldots, t_{i-1}, A_{i}^{ \pm}, t_{i}, \ldots, t_{p}\right) \in \mathbb{R}^{p+1} \mid\right. \\
A_{j}^{-} & \left.\leq t_{j}^{\prime} \leq A_{j}^{+}, 1 \leq t_{j}^{\prime} \leq i-1 \& A_{j+1}^{-} \leq t_{j+1}^{\prime} \leq A_{j+1}^{+} i \leq j \leq p\right\},
\end{aligned}
$$

is the face $\left\{t_{i}^{\prime}=A_{i}^{ \pm}\right\}$of $J^{p+1}$. The geometric boundary of the $p+1$-cube $J^{p+1}$ is

$$
\partial J^{p+1}=\bigcup_{1 \leq i \leq p}(-1)^{i+1}\left[\phi_{i}^{+}\left(J_{i}^{p+1}\right)-\phi_{i}^{-}\left(J_{i}^{p+1}\right)\right]
$$


which is the union of appropriately signed faces of $J^{p+1}$.

Let $\sigma: J^{p+1} \rightarrow S$ be a singular $p+1$-cube, that is, $\sigma \in K_{p+1}(\mathbf{S})$. Define the boundary operator $\partial: K_{p+1}(\mathbf{S}) \rightarrow K_{p}(\mathbf{S})$ by

$$
\partial \sigma=\sum_{i=1}^{p}(-1)^{i+1}\left[\sigma \circ \phi_{i}^{+}-\sigma \circ \phi_{i}^{-}\right] .
$$

For every $\left(t_{1}, \ldots, t_{p}\right) \in \mathbb{R}^{p}$ we have

$$
\begin{gathered}
\partial \sigma\left(t_{1}, \ldots, t_{p}\right)=\sum_{i=1}^{p}(-1)^{i+1}\left[\sigma\left(t_{1}, \ldots, t_{i-1}, A_{i}^{+}, t_{i}, \ldots, t_{p}\right)\right. \\
\left.-\sigma\left(t_{1}, \ldots, t_{i-1}, A_{i}^{-}, t_{i}, \ldots, t_{p}\right)\right] .
\end{gathered}
$$

So

$$
\left.\partial \sigma\right|_{\partial J^{p+1}}=\sum_{i=1}^{p}(-1)^{i+1}\left[\sigma \circ \phi_{i}^{+}\left(J_{i}^{p+1}\right)-\sigma \circ \phi_{i}^{-}\left(J_{i}^{p+1}\right)\right] .
$$

Since

$$
\partial J^{p+1}=\bigcup_{1 \leq i \leq p}\left((-1)^{i+1} \phi_{i}^{+}\left(J_{i}^{p+1}\right) \cup(-1)^{i} \phi_{i}^{-}\left(J_{i}^{p+1}\right)\right),
$$

the map $\left.\partial \sigma\right|_{\partial J^{p+1}}: \partial J^{p+1} \rightarrow S$ is the sum of the signed singular $p$-cubes $\sigma_{i}^{ \pm}: \phi_{i}^{ \pm}\left(J_{i}^{p+1}\right) \rightarrow S$ for $1 \leq i \leq p$, in $C_{p}(\mathbf{S})$. Hence $\partial \sigma: \partial J^{p+1} \rightarrow S$ is a singular $p$-chain in $K_{p}(\mathbf{S})$. Thus the boundary operator $\partial$ is well defined. When $p=0$, then $\sigma \circ \phi_{i}^{ \pm}$are the image under $\sigma$ of the end points of $J^{1}$. The boundary operator $\partial$ is a group homomorphism because it maps a generator of the group $K_{p+1}(\mathbf{S})$ into an element of $K_{p}(\mathbf{S})$.

Lemma 5.3.2 $\partial^{2}=0$.

Proof. Using the definition of the boundary operator $\partial$, we get

$$
\begin{gathered}
\partial(\partial \sigma)\left(t_{1}, \ldots, t_{p}\right)=\sum_{j=1}^{p}(-1)^{j+1}\left[\partial \sigma\left(t_{1}, \ldots, t_{j-1}, A_{j}^{+}, t_{j}, \ldots, t_{p}\right)\right. \\
\left.-\partial \sigma\left(t_{1}, \ldots, t_{j-1}, A_{j}^{-}, t_{j}, \ldots, t_{p}\right)\right] \\
=\sum_{j=1}^{p}\left[\sum _ { a = \{ + , - \} } \sum _ { i = 1 } ^ { j } a ( - 1 ) ^ { i + j } \left[\sigma\left(t_{1}, \ldots, t_{i-1}, A_{i}^{|a|}, t_{i}, \ldots, t_{j-1}, A_{j}^{a}, t_{j}, \ldots, t_{p}\right)\right.\right. \\
\left.-\sigma\left(t_{1}, \ldots, t_{i-1}, A_{i}^{-|a|}, t_{i+1}, \ldots, t_{j-1}, A_{j}^{a}, t_{j}, \ldots, t_{p}\right)\right] \\
+\sum_{i=j}^{p}(-1)^{i}\left[\sigma\left(t_{1}, \ldots, t_{j-1}, A_{j}^{a}, t_{j}, \ldots, t_{i-1}, A_{i}^{|a|}, t_{i}, \ldots, t_{p}\right)\right. \\
\left.\left.-\sigma\left(t_{1}, \ldots, t_{j-1}, A_{j}^{a}, t_{j}, \ldots, t_{i-1}, A_{i}^{-|a|}, t_{i}, \ldots, t_{p}\right)\right]\right]
\end{gathered}
$$


where $|a|=+$ if $a=+$ or - . The terms in the above expression, where the superscripts of $A_{i}^{|a|}$ and $A_{j}^{a}$ are the same sign, cancel. We check this for $A_{i}^{+}$and $A_{j}^{+}$. We look at

$$
\begin{aligned}
& \sum_{j=1}^{p} \sum_{i=1}^{j}(-1)^{i+j} \sigma\left(t_{1}, \ldots, t_{i-1}, A_{i}^{+}, t_{i}, \ldots, t_{j-1}, A_{j}^{+}, t_{j}, \ldots, t_{p}\right) \\
& -\sum_{j=1}^{p} \sum_{i=j}^{p}(-1)^{i+j} \sigma\left(t_{1}, \ldots, t_{j-1}, A_{j}^{+}, t_{j}, \ldots, t_{i-1}, A_{i}^{+}, t_{i}, \ldots, t_{p}\right)
\end{aligned}
$$

Interchanging the indices, the second sum in (20) reads

$$
-\sum_{j=1}^{p} \sum_{i=1}^{j}(-1)^{i+j} \sigma\left(t_{1}, \ldots, t_{i-1}, A_{i}^{+}, t_{i}, \ldots, t_{j-1}, A_{j}^{+}, t_{j}, \ldots, t_{p}\right),
$$

which cancels the first sum in (20).

Let $\sigma: J^{p} \rightarrow S$ be an element of $C_{p}(\mathbf{S})$ with $p>0$. Define

$$
K(\sigma): I \times J^{p} \rightarrow I \times S:\left(t_{1}^{\prime}, t_{2}^{\prime}, \ldots, t_{p+1}^{\prime}\right) \mapsto\left(t_{1}^{\prime}, \sigma\left(t_{2}^{\prime}, \ldots, t_{p+1}^{\prime}\right)\right) .
$$

Then for $p>0$ we obtain

$$
K: K_{p}(\mathbf{S}) \rightarrow K_{p+1}(\mathbf{I} \times \mathbf{S}): \sigma \mapsto K(\sigma) .
$$

When $p=0$ we have $K(\sigma)\left(t_{1}\right)=\left(t_{1}, \sigma\right)$, where $\sigma \in K_{0}(\mathbf{S})=S$. For $i=0,1$ define $u_{i}: S \rightarrow I \times S: x \mapsto(i, x)$.

Lemma 5.3.3. We have

$$
K \partial+\partial K=\left(u_{1}\right)_{*}-\left(u_{0}\right)_{*},
$$

where $\left(u_{i}\right)_{*}: K_{p}(\mathbf{S}) \rightarrow K_{p+1}(\mathbf{I} \times \mathbf{S})$ is the chain map induced by $u_{i}$. When $p=0$ equation $(19)_{p}$ reads

$$
K \partial=\left(u_{1}\right)_{*}-\left(u_{0}\right)_{*} .
$$

Proof. Let $\sigma: J^{p} \rightarrow S$ be a singular $p$-cube represented by $f \in \mathcal{F}(\mathbf{S})$. Then

$$
\begin{aligned}
& K \partial \sigma\left(t_{1}, \ldots, t_{p}\right)+\partial K \sigma\left(t_{1}, \ldots, t_{p}\right)= \\
& \quad=K\left(\sum_{i=1}^{p}(-1)^{i+1}\left[\sigma\left(t_{1}, \ldots, t_{i-1}, A_{i}^{+}, t_{i}, \ldots, t_{p}\right)-\sigma\left(t_{1}, \ldots, t_{i-1}, A_{i}^{-}, t_{i}, \ldots, t_{p}\right)\right]\right. \\
& \left.\quad+\partial\left(t_{0}, \sigma\left(t_{1}, \ldots, t_{p}\right)\right)\right)
\end{aligned}
$$




$$
\begin{aligned}
=\sum_{i=1}^{p}(-1)^{i+1}\left[\left(t_{0}, \sigma\left(t_{1}, \ldots, t_{i-1}, A_{i}^{+}, t_{i}, \ldots, t_{p}\right)\right)\right. & \left.-\left(t_{0}, \sigma\left(t_{1}, \ldots, t_{i-1}, A_{i}^{-}, t_{i}, \ldots, t_{p}\right)\right)\right]+\left(1, \sigma\left(t_{1}, \ldots, t_{p}\right)\right) \\
& \quad-\sum_{i=1}^{p}(-1)^{i+1}\left[\left(t_{0}, \sigma\left(t_{1}, \ldots, t_{i-1}, A_{i}^{+}, t_{i}, \ldots, t_{p}\right)\right)\right. \\
& \left.\quad-\left(t_{0}, \sigma\left(t_{1}, \ldots, t_{i-1}, A_{i}^{-}, t_{i}, \ldots, t_{p}\right)\right)\right]-\left(0, \sigma\left(t_{1}, \ldots, t_{p}\right)\right) \\
= & \left(1, \sigma\left(t_{1}, \ldots, t_{p}\right)\right)-\left(0, \sigma\left(t_{1}, \ldots, t_{p}\right)\right) \\
= & \left(u_{1}\right)_{*} \sigma\left(t_{1}, \ldots, t_{p}\right)-\left(u_{0}\right)_{*} \sigma\left(t_{1}, \ldots, t_{p}\right) .
\end{aligned}
$$

The set $K_{p}(\mathbf{S})$ of singular $p$-chains on $\mathbf{S}$, being the free abelian group generated by $C_{p}(\mathbf{S})$, the set of singular $p$-cubes on $\mathbf{S}$, may be considered as the set of vectors of a real vector space. The set $K^{p}(\mathbf{S})$ of singular $p$-cochains on $\mathbf{S}$ is the dual $K_{p}(\mathbf{S})^{*}$ of the vector space $K_{p}(\mathbf{S})$, because any function $f: K_{p}(\mathbf{S}) \rightarrow \mathbb{R}$ on the generators in $K_{p}(\mathbf{S})$ extends to a unique real linear function on the real vector space $K_{p}(\mathbf{S})$.

Lemma 5.3.4 The dual operator

$$
K^{*}: K^{p+1}(\mathbf{S}) \rightarrow K^{p}(\mathbf{S}): \alpha \mapsto \alpha_{\circ} K .
$$

sends $\bar{G}^{p+1}(\mathbf{I} \times \mathbf{S})$ to $G^{p}(\mathbf{S})$.

Proof. Every $f \in \mathcal{F}_{\mathbf{I} \times \mathbf{S}}$ can be represented as $f=\phi\left(t, f_{1}, \ldots, f_{p}\right)$, where $t \in I$ and $f_{i} \in \mathcal{F}_{\mathbf{S}}$. Every element $\omega$ of $\bar{G}^{p+1}(\mathbf{I} \times \mathbf{S})$ is a sum of terms of the form 1: $a \mathrm{~d} t \wedge \mathrm{d} f_{1} \wedge \cdots \wedge \mathrm{d} f_{p}$ or 2: $a \mathrm{~d} f_{1} \wedge \cdots \wedge \mathrm{d} f_{p+1}$, where $a \in \overline{\mathcal{F}}_{\mathbf{I} \times \mathbf{S}}$ and $f_{j} \in \mathcal{F}_{\mathbf{S}}$. Let $\sigma: I \times J^{p} \rightarrow X$ be a singular $p+1$-cube in $S$ represented by $g: U \rightarrow S$ in $\mathcal{F}_{\mathbf{S}}(U)$. Set $b=a_{\circ} g$ and $g_{j}=f_{j} \circ g$. Suppose that $\omega$ has the form in item 2. Then $K^{*} \omega=0$. This follows because each $g_{j}$ is independent of $t$ and thus $\frac{\partial\left(g_{1}, \ldots, g_{p+1}\right)}{\partial\left(t, t_{1}, \ldots, t_{p}\right)}=0$. When $\omega$ has the form in item 1 , then

$$
\begin{aligned}
K^{*} \omega(\sigma) & =\int_{I \times J^{p}} b \frac{\partial\left(g_{1}, \ldots, g_{p}\right)}{\partial\left(t_{1}, \ldots, t_{p}\right)} \mathrm{d} t \mathrm{~d} t_{1} \cdots \mathrm{d} t_{p} \\
& =\int_{I} b \mathrm{~d} t \int_{J^{p}} \frac{\partial\left(g_{1}, \ldots, g_{p}\right)}{\partial\left(t_{1}, \ldots, t_{p}\right)} \mathrm{d} t_{1} \cdots \mathrm{d} t_{p+1} .
\end{aligned}
$$

Consider $\int_{I} b \mathrm{~d} t$ as a function $f_{0}: S \rightarrow \mathbb{R}$. Since $a \in \overline{\mathcal{F}}_{\mathbf{I} \times \mathbf{S}}$, it follows that $f_{0}=\psi\left(f_{1}^{\prime}, \ldots, f_{p}^{\prime}\right)$ for some $f_{j}^{\prime} \in \mathcal{F}_{\mathbf{S}}$ and some $\psi \in C^{\infty}\left(\mathbb{R}^{p}\right)$. Thus $f_{0} \in \mathcal{F}_{\mathbf{S}}$. Since $\frac{\partial\left(g_{1}, \ldots, g_{p}\right)}{\partial\left(t_{1}, \ldots, t_{p}\right)}$ does not depend on $t$, it follows that $K^{*} \omega=f_{0} \mathrm{~d} f_{1} \wedge \cdots \wedge \mathrm{d} f_{p}$.

Lemma 5.3.5 For every $\mathbf{S} \in \mathcal{D}$, the dual $\partial^{*}$ of the boundary operator $\partial$ : $K_{p+1}(\mathbf{S}) \rightarrow K_{p}(\mathbf{S})$ is the exterior derivative d: $G^{p}(\mathbf{S}) \rightarrow G^{p+1}(\mathbf{S})$.

Proof. Let $\sigma: J^{p+1} \rightarrow S$ be a singular $p+1$ cube represented by $f \in \mathcal{F}_{\mathbf{S}}(U)$. 
By definition, for $\alpha \in K_{p}(\mathbf{S})^{*}=K^{p}(\mathbf{S})$ we have

$$
\begin{aligned}
& \alpha(\partial \sigma)= \lambda_{p}\left(f_{0}, \ldots, f_{p}\right) \partial \sigma, \quad \text { where } f_{i} \in \mathcal{F}_{\mathbf{S}} \\
&= \lambda_{p}\left(f_{0}, \ldots, f_{p}\right)\left(\sum_{i=1}^{p}(-1)^{i+1}\left[\sigma \circ \phi_{i}^{+}\left(J_{i}^{p+1}\right)-\sigma \circ \phi_{i}^{-}\left(J_{i}^{p+1}\right)\right)\right. \\
& \quad \text { since } \partial \sigma: \partial J^{p+1} \rightarrow S \text { is a } p \text {-chain } \\
&=\sum_{i=1}^{p}(-1)^{i+1} \int_{\phi_{i}^{+}\left(J_{i}^{p+1}\right)-\phi_{i}^{-}\left(J_{i}^{p+1}\right)} g_{0} \mathrm{~d} g_{1} \wedge \cdots \wedge \mathrm{d} g_{p}, \\
& \quad \text { where } \lambda_{p}\left(f_{0}, \ldots, f_{p}\right)\left(\sigma \circ \phi_{i}^{ \pm}\left(J_{i}^{p+1}\right)\right)= \\
& \quad=\int_{\phi_{i}^{ \pm}\left(J_{i}^{p+1}\right)} g_{0} \mathrm{~d} g_{1} \wedge \cdots \wedge \mathrm{d} g_{p} \text { and } g_{i}= \\
&=\int_{\sum_{i} \circ f}(-1)^{i+1}\left[\phi_{i}^{+}\left(J_{i}^{p+1}\right)-\phi_{i}^{-}\left(J_{i}^{p+1}\right)\right] \\
&=\int_{\partial J^{p+1}} g_{0} \mathrm{~d} g_{1} \wedge \cdots \wedge \text { is represented by } f \\
&=\int_{J^{p+1}} \mathrm{~d}\left(g_{0} \wedge \mathrm{d} g_{1} \wedge \cdots \wedge \mathrm{d} g_{p}\right. \\
&=\lambda_{p+1}\left(\mathbf{1}, f_{0}, \ldots, f_{p}\right) \sigma=\mathrm{d} \lambda_{p}\left(f_{0}, \ldots, f_{p}\right) \sigma=\mathrm{d} \alpha(\sigma)
\end{aligned}
$$

This implies $\partial^{*}=\mathrm{d}$ because $\left(\partial^{*} \alpha\right) \sigma=\alpha(\partial \sigma)=\mathrm{d} \alpha(\sigma)$ for every $\sigma \in C^{p}(\mathbf{S})$.

We now finish the proof of the Poincaré lemma proposition 5.3.1.

The real vector subspace $\bar{G}(\mathbf{I} \times \mathbf{S})$ of $G(\mathbf{I} \times \mathbf{S})$ is invariant under d Hence the operator $(K \partial+\partial K)^{*}=\mathrm{d} K^{*}+K^{*}$ d sends $\bar{G}^{p+1}(\mathbf{I} \times \mathbf{S})$ to $G^{p+1}(\mathbf{S})$. From equation $(19)_{p}$ we obtain

$$
\mathrm{d} K^{*}+K^{*} \mathrm{~d}=u_{1}^{*}-u_{0}^{*}
$$

on $\bar{G}^{p+1}(\mathbf{S})$. When $p=0$ equation $(20)_{p}$ reads

$$
K^{*} \mathrm{~d}=u_{1}^{*}-u_{0}^{*} .
$$

Let $h: I \times S \rightarrow S$ be a differentiable contraction of $S$. For $\alpha \in G^{p+1}(\mathbf{S})$ with $\mathrm{d} \alpha=0$ set $\omega=h^{*} \alpha$. Since $h$ is a well behaved differentiable map, it follows that $\omega \in \bar{G}^{p+1}(\mathbf{I} \times \mathbf{S})$. Moreover, $\mathrm{d} \omega=\mathrm{d} h^{*} \alpha=h^{*} \mathrm{~d} \alpha=0$ because d commutes with $h^{*}$. From $h \circ u_{1}=\mathrm{id}_{S}$, we get $u_{1}^{*} \omega=\alpha$. Since $h \circ u_{0}$ is a constant map, we obtain $u_{0}^{*} \omega=0$. Thus equation $(20)_{p}$ gives

$$
\alpha=u_{1}^{*} h^{*} \alpha-u_{0}^{*} h^{*} \alpha=\mathrm{d}\left(K^{*} \omega\right)+K^{*} \mathrm{~d} \omega=\mathrm{d}\left(K^{*} \omega\right),
$$

using $\mathrm{d} \omega=0$. Setting $\beta=K^{*} \omega$ proves proposition 5.3.5.

Corollary 5.3.5A Suppose that the differentiable space $\mathbf{S}$ is differentiably contractible and $f \in \mathcal{F}_{\mathbf{S}}$ such that $\mathrm{d} f=0$. Then $f$ is a constant function. 
Proof. This follows immediately from equation $(20)_{0}$ for

$$
u_{1}^{*} f-u_{0}^{*} f=K^{*}(\mathrm{~d} f)=0 .
$$

But $u_{1}^{*} f=f$ and $u_{0}^{*} f$ is a constant function on $S$.

\subsection{Sheaf of differential forms}

Consider the differentiable space $\mathbf{S}=(S, \mathcal{F})$. Let $U$ be an open subset of $S$. Let $\sigma: J^{p} \rightarrow S$ be a singular $p$-cube on $\mathbf{S}$ represented by $f \in \mathcal{F}_{\mathbf{S}}$. Suppose that $\sigma\left(J^{p}\right) \subseteq U$. Then $\sigma_{U}: J^{p} \rightarrow U: \mathbf{t} \mapsto \sigma(\mathbf{t})$ is a singular $p$ cube on $U$ represented by $\left.f\right|_{U} \in \mathcal{F}_{U}$. Let $C_{p}(U)$ be the set of all singular $p$-cubes on $U$. Let $C^{p}(U)$ be the set of all functions $\left.\alpha\right|_{U}: C_{p}(U) \rightarrow \mathbb{R}$ such that $\alpha: C_{p}(\mathbf{S}) \rightarrow \mathbb{R}$ is a singular $p$-cube on $\mathbf{S}$. For every $p>0$ define the mapping

$$
\left(\lambda_{p}\right)_{U}: \mathcal{F}_{U}^{p+1} \rightarrow C^{p}(U):\left.\left(\left.f_{0}\right|_{U}, \ldots,\left.f_{p}\right|_{U}\right) \rightarrow \lambda_{p}\right|_{U}\left(\left.f_{0}\right|_{U}, \ldots,\left.f_{p}\right|_{U}\right),
$$

where $g_{i}=\left.\left.f\right|_{U} \circ f_{i}\right|_{U} \in \mathcal{F}_{U}$ and

$$
\left(\lambda_{p}\right)_{U}\left(\left.f_{0}\right|_{U}, \ldots, f_{p} \mid U\right)\left(\sigma_{U}\right)=\int_{J^{p}} g_{0} \frac{\partial\left(g_{1}, \ldots, g_{p}\right)}{\partial\left(t_{1}, \ldots, t_{p}\right)} \mathrm{d} t_{1} \cdots \mathrm{d} t_{p} .
$$

When $p=0$ then $C^{0}(U)=\left.\mathcal{F}\right|_{U}$. Let $G^{0}(U)$ be the vector space generated by the image of $\left.\lambda_{p}\right|_{U}$. If $F: \mathbf{S} \rightarrow \mathbf{S}^{\prime}$ is a differentiable maping such that $U=F^{-1}\left(U^{\prime}\right)$, where $U^{\prime}$ is an open subset of $\mathbf{S}^{\prime}$ and $U$ is an open subset of $\mathbf{S}$, then $F$ induces a real linear mapping

$$
F^{*}: C^{p}\left(U^{\prime}\right) \rightarrow C^{p}(U):\left(\lambda_{p}\right)_{U^{\prime}}\left(f_{0}^{\prime}, \ldots, f_{p}^{\prime}\right) \mapsto\left(\lambda_{p}\right)_{U}\left(f_{0}, \ldots, f_{p}\right),
$$

where $f_{i}^{\prime}=f_{i} \circ F \in \mathcal{F}_{U^{\prime}}$ since $f_{i} \in \mathcal{F}_{U}$. Hence $F^{*}: G^{p}\left(U^{\prime}\right) \rightarrow G^{p}(U)$. Let $\mathcal{G}^{p}$ be the local category whose objects are the spaces $G^{p}(U)$ for $U$ an open subset of $\mathbf{S}$ and whose maps are real linear mappings $F^{*}: G^{p}\left(U^{\prime}\right) \rightarrow G^{p}(U)$ induced by differentiable mappings $F_{U}: U \rightarrow U^{\prime}$. Thus $G: \mathcal{D} \rightarrow \mathcal{G}^{p}$ is a contravariant functor from the local category $\mathcal{D}$ of differentiable spaces and differentiable maps to the local category $\mathcal{G}^{p}$ of differential $p$ forms on a differentiable space and real linear maps between spaces of differential $p$-forms. For every object $\mathbf{S}$ in the local category $\mathcal{D}$ we have a covariant functor $S_{\mathbf{S}}: \mathcal{C}^{0} \rightarrow \mathcal{G}^{p}$ whose value at the object $\mathbf{S}_{U}$ is the open subset $U$ of $S$ and whose value at the inclusion map $\iota_{U} \mid V: V \subseteq U \rightarrow S$ is the map $\left(\left.\iota_{U}\right|_{V}\right)^{*}: G^{p}(V) \rightarrow G^{p}(U)$. For every $\mathbf{S} \in \mathcal{D}$ the map

$$
G^{p}=G_{\circ} S_{\mathbf{S}}: \mathcal{C}^{0} \rightarrow \mathcal{G}^{p}: U \subseteq S \rightarrow G^{p}(U)
$$

is the sheaf of differential $p$ forms on $\mathbf{S}$. Note that the sheaf $G^{0}$ is equal to the sheaf $F^{0}$.

\section{5 de Rham's theorem}

In this subsection we prove Smith's version of de Rham's theorem for differentiable spaces, see [13]. 
We now assume that the differentiable space $\mathbf{S}$ is locally differentiably contractible, that is, for every $x \in S$ there is open subset $U$ of $S$ containing $x$ such that the differential space $\left(U, C^{\infty}(U)\right)$ is contractible. We have the sheaf homomorphism d: $G^{p} \rightarrow G^{p+1}$ induced by the exterior derivative. Its kernel defines the sheaf $Z^{p}: \mathcal{C}^{0} \rightarrow \mathcal{G}^{p}: U \mapsto G^{p}(U)$ of closed $p$-forms on $S$.

Lemma 5.5.1 The sheaf $Z^{0}$ is the sheaf $R$ of locally constant real valued functions on $\mathbf{S}$.

Proof. Because $\mathbf{S}$ is locally differentiably contractible, from corollary $5.3 .5 \mathrm{~A}$ it follows that if $\mathrm{d} f=0$ on a contractible (and hence connected) open subset $U$ of $\mathbf{S}$, then $f$ is constant on $U$.

Hence we have an exact sequence of sheaves

$$
0 \longrightarrow Z^{q} \stackrel{\iota}{\longrightarrow} \Lambda^{q} \stackrel{\mathrm{d}}{\longrightarrow} Z^{q+1} \longrightarrow 0
$$

for $q \geq 0$. Here $\Lambda^{q}: \mathcal{C}^{0} \rightarrow \mathcal{G}^{p}: U \mapsto \Lambda^{p}(U)$ is the sheaf of differential $q$-forms on $\mathbf{S}$ generated by $f_{i} \in \mathcal{F}_{U}$, where $U$ is an open subset of $\mathbf{S}$. In other words, $\alpha \in \Lambda^{p}(U)$ if and only if $\alpha=f_{0} \mathrm{~d} f_{1} \wedge \cdots \wedge \mathrm{d} f_{p}$, where $f_{i} \in \mathcal{F}_{U}$.

Let $\mathbf{S} \in \mathcal{D}$. Suppose that $S$ is a paracompact topological space. Then there is a locally finite good covering $\mathcal{U}=\left\{U_{i}\right\}_{i \in I}$ of $S$, that is, every finite intersection of elements of $\mathcal{U}$ is either contractible or empty. Consider the sheaf $F^{0}: \mathcal{C}^{0} \rightarrow \mathcal{F}^{0}: U \mapsto \mathcal{F}_{U}$, where $\mathcal{F}^{0}$ is the category, for which an object is the space $\mathcal{F}_{U}$ of differentiable functions on the differentiable space $\mathbf{S}_{U}=\left(U, \mathcal{F}_{U}\right)$ with $U$ being an open subset of $S$, and a map is

$$
\left(\left.\iota_{U}\right|_{V}\right)^{*}: \mathcal{F}_{V} \rightarrow \mathcal{F}_{U}: f \mapsto f \circ\left(\left.\iota_{U}\right|_{V}\right),
$$

We suppose that the sheaf $F^{0}$ is soft, that is, for every closed subset $C$ of $S$ and every $g \in \mathcal{F}_{C}=\left.\mathcal{F}_{\mathbf{S}}\right|_{C}$ there is an $f \in \mathcal{F}_{\mathbf{S}}$ such that $\left.f\right|_{C}=g$. We have

Lemma 5.5.2 Let $\mathcal{S}=\left(S, \mathcal{F}_{U}\right)$ be a differentiable space. The sheaf $\Lambda^{q}$ of differential forms generated by $\mathcal{F}_{U}$ and exterior derivative of elements of $\mathcal{F}_{U}$ for every open subset $U$ of $S$ is soft.

Proof. Let $\alpha=f_{0} \mathrm{~d} f_{1} \wedge \cdots \wedge \mathrm{d} f_{p} \in \Lambda^{p}(C)$, where $f_{i} \in \mathcal{F}_{C}$ for some closed subset $C$ of $S$. Because the sheaf $F^{0}$ is soft, for each $f_{i}$ there is a $g_{i} \in \mathcal{F}_{\mathbf{S}}$ such that $\left.g_{i}\right|_{C}=f_{i}$. Thus $\beta=g_{0} \wedge \mathrm{d} g_{1} \wedge \cdots \wedge \mathrm{d} g_{p}$ is a $p$-form on $S$, namely, $\beta \in \Lambda^{p}(S)$, such that $\left.\beta\right|_{C}=\alpha$. Hence the sheaf $\Lambda^{p}$ is soft.

We now review the definition of the $q^{\text {th }}$ cohomology group $\mathrm{H}^{q}\left(\mathcal{U}, \Lambda^{q}\right)$ of the good covering $\mathcal{U}$ of $S$ having values in the sheaf $\Lambda^{q}$, see Lukina, et al. 9. Recall that a $q$-cochain is an alternating map

$$
f: I^{q+1} \rightarrow \Lambda^{q}\left(U_{i_{0}} \cap \cdots \cap U_{i_{q}}\right):\left(i_{0}, \ldots, i_{q}\right) \mapsto f\left(i_{0}, \ldots, i_{q}\right),
$$

where $U_{i_{j}} \in \mathcal{U}$ for $j=0, \ldots, q$. The set $C^{q}\left(\mathcal{U}, \Lambda^{q}\right)$ of $q$-cochains is a group. The $q$-coboundary homomorphism is the map

$$
\begin{aligned}
\delta^{q}: & C^{q}\left(\mathcal{U}, \Lambda^{q}\right) \rightarrow C^{q+1}\left(\mathcal{U}, \Lambda^{q}\right): f \mapsto\left(\delta^{q} f\right)\left(i_{0}, \ldots, i_{q+1}\right)= \\
& =\left.\sum_{j=0}^{q+1} \iota_{U_{i_{0}} \cap \cdots \cap \widehat{U}_{i_{j}} \cap \cdots \cap U_{i_{q+1}}}\right|_{U_{i_{0}} \cap \cdots \cap U_{i_{q+1}}}\left(f\left(i_{0}, \ldots, i_{j-1}, \widehat{i_{j}}, i_{j+1}, \ldots, i_{q+1}\right)\right) .
\end{aligned}
$$


It is straightforward to show that $\delta^{q+1} \circ \delta^{q}=0$. We will omit the superscipt and write $\delta$ instead of $\delta^{q}$ when there is no risk of confusion. An element of $\operatorname{ker} \delta^{q}$ is called a $q$-cocycle. The set of $q$-cocycles forms a subgroup of $C^{q}\left(\mathcal{U}, \Lambda^{q}\right)$. The group $\mathrm{H}^{q}\left(\mathcal{U}, \Lambda^{q}\right)=\operatorname{ker} \delta^{q} /$ image $\delta^{q-1}$, when $q \geq 1$ and $\mathrm{H}^{0}\left(\mathcal{U}, \Lambda^{q}\right)=\operatorname{ker} \delta^{0}$, when $q=0$, is the $q^{\text {th }}$ cohomology group of the open covering $\mathcal{U}$ with values in the sheaf $\Lambda^{q}$. Note that $\mathrm{H}^{0}\left(\mathcal{U}, \Lambda^{q}\right)=Z^{q}(\mathbf{S})$.

The following diagram of sheaf cochain complexes is exact.

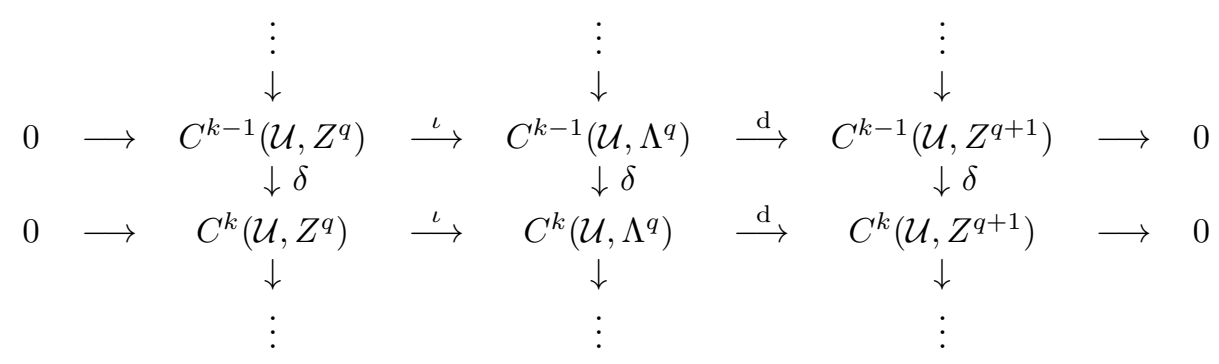

where $\delta$ is the coboundary operator, $\iota$ is the inclusion, and d the exterior derivative. The above diagram of cochain complexes gives rise to the long exact sequence of cohomology groups

$$
0 \rightarrow \mathrm{H}^{0}\left(\mathcal{U}, Z^{q}\right) \stackrel{\iota_{*}}{\longrightarrow} \mathrm{H}^{0}\left(\mathcal{U}, \Lambda^{q}\right) \stackrel{\mathrm{d}_{*}}{\longrightarrow} \mathrm{H}^{0}\left(\mathcal{U}, Z^{q+1}\right) \stackrel{\delta_{*}}{\longrightarrow} \mathrm{H}^{1}\left(\mathcal{U}, Z^{q}\right) \rightarrow \cdots
$$

where $\iota_{*}, \mathrm{~d}_{*}$, and $\delta_{*}$ are cohomology homomorphisms induced by $\iota, \mathrm{d}$, and $\delta$ respectively. Because the sheaf $\Lambda^{q}$ is soft, its $q^{\text {th }}$ cohomology group vanishes when $q \geq 1$. Thus the long exact sequence (22) falls apart into the exact sequences

$$
0 \rightarrow \mathrm{H}^{0}\left(\mathcal{U}, Z^{q}\right) \stackrel{\iota_{*}}{\longrightarrow} \mathrm{H}^{0}\left(\mathcal{U}, \Lambda^{q}\right) \stackrel{\mathrm{d}_{*}}{\longrightarrow} \mathrm{H}^{0}\left(\mathcal{U}, Z^{q+1}\right) \stackrel{\delta_{*}}{\longrightarrow} \mathrm{H}^{1}\left(\mathcal{U}, Z^{q}\right) \rightarrow 0
$$

and

$$
0 \rightarrow \mathrm{H}^{k}\left(\mathcal{U}, Z^{q+1}\right) \stackrel{\delta_{*}}{\longrightarrow} \mathrm{H}^{k+1}\left(\mathcal{U}, Z^{q}\right) \rightarrow 0,
$$

for every $k \geq 1$. Now $\mathrm{H}^{0}(\mathcal{U}, R)=Z^{0}(\mathbf{S})$. Applying (24) iteratively gives

$$
\mathrm{H}^{q}(\mathcal{U}, R)=\mathrm{H}^{q}\left(\mathcal{U}, Z^{0}\right) \simeq \mathrm{H}^{q-1}\left(\mathcal{U}, Z^{1}\right) \simeq \cdots \simeq \mathrm{H}^{1}\left(\mathcal{U}, Z^{q-1}\right),
$$

where $\simeq$ means isomorphic to. For $q \geq 1$ from (23) we find that

$$
\begin{aligned}
\mathrm{H}^{1}\left(\mathcal{U}, Z^{q-1}\right) & \simeq \mathrm{H}^{0}\left(\mathcal{U}, Z^{q}\right) /\left.\operatorname{ker} \delta_{*}\right|_{\mathrm{H}^{0}\left(\mathcal{U}, Z^{q}\right)} \\
& \simeq \mathrm{H}^{0}\left(\mathcal{U}, Z^{q}\right) / \mathrm{d}_{*}\left(\mathrm{H}^{0}\left(\mathcal{U}, \Lambda^{q-1}\right)\right) \simeq Z^{q}(\mathbf{S}) / \mathrm{d} \Lambda^{q-1}(\mathbf{S}) .
\end{aligned}
$$

Hence we obtain Smith's version of de Rham's theorem for a differential space.

Theorem 5.5.3 Let $\mathbf{S}=\left(S, \mathcal{F}_{\mathbf{S}}\right)$ be a differentiable space, where $S$ is locally differentiably contractible and paracompact. Moreover, suppose that the sheaf $F^{0}: \mathcal{C}^{0} \rightarrow \mathcal{F}^{0}: U \mapsto \mathcal{F}_{U}$ is soft. Then for every $q \geq 1$ 


$$
\mathrm{H}^{q}(\mathcal{U}, R)=\mathrm{H}^{q}\left(\mathcal{U}, Z^{0}\right) \simeq Z^{q}(\mathbf{S}) / \mathrm{d} \Lambda^{q-1}(\mathbf{S})=\mathrm{H}_{\mathrm{dR}}^{q}(\mathbf{S})
$$

and for $q=0$

$$
\mathrm{H}^{0}(\mathcal{U}, R)=Z^{0}(\mathbf{S})=R=\mathrm{H}_{\mathrm{dR}}^{0}(\mathbf{S}) .
$$

Corollary 5.5.3A For $q \in \mathbb{Z}_{\geq 0}$ the $q^{\text {th }}$ Čech sheaf cohomology group $\check{H}^{q}(\mathbf{S}, R)$ of $\mathbf{S}$ with values in the sheaf $R$ of locally constant real valued functions on $\mathbf{S}$ is isomorphic to the $q^{\text {th }}$ de Rham cohomology group $\mathrm{H}_{\mathrm{dR}}^{q}(\mathbf{S})$ of $\mathbf{S}$.

Proof. From equations $(23)_{q}$ and $(23)_{0}$ it follows that $\mathrm{H}^{q}(\mathcal{U}, R) \simeq \mathrm{H}_{\mathrm{dR}}^{q}(\mathbf{S})$, which is independent of the good covering $\mathcal{U}$ of $\mathbf{S}$. By definition $\check{H}^{q}(\mathbf{S}, R)=$ $\mathrm{H}^{q}(\mathcal{U}, R)$.

\section{The orbit space of a proper action}

Let $\Phi: G \times M \rightarrow M:(g, m) \mapsto \Phi_{g}(m)=g \cdot m$ be a proper action of a Lie group $G$ on a smooth manifold $M$. We denote the space of smooth $G$-invariant functions on $M$ by $C^{\infty}(M)^{G}$. Let $M / G=\{G \cdot m \mid m \in M\}=\bar{M}$ be the space of $G$-orbits in $M$ with $G$-orbit map $\pi: M \rightarrow \bar{M}: m \mapsto \bar{m}=G \cdot m$. The set $C^{\infty}(\bar{M})=\left\{f: \bar{M} \rightarrow \mathbb{R} \mid \pi^{*} f \in C^{\infty}(M)^{G}\right\}$ is a differential structure on $\bar{M}$. The differential space $\left(\bar{M}, C^{\infty}(\bar{M})\right)$ is subcartesian and is locally closed. Moreover, the differential space topology on $\bar{M}$ is equivalent to the quotient topology. So $\bar{M}$ is paracompact.

\subsection{Vector fields on $\bar{M}$}

Let $X$ be a $G$-invariant vector field on $M$, that is, $\Phi_{g}^{*} X(m)=T_{m} \Phi_{g^{-1}} X\left(\Phi_{g}(m)\right)$ $=X(m)$ for every $g \in G$ and every $m \in M$.

Lemma 6.1.1 If $X$ is a $G$-invariant vector field on $M$, then

$$
X: C^{\infty}(M)^{G} \rightarrow C^{\infty}(M)^{G}: f \mapsto X(f)=L_{X} f
$$

is a derivation of $C^{\infty}(M)^{G}$.

Proof. $X$ maps $C^{\infty}(M)^{G}$ into itself, because for every $g \in G$ and every $f \in$ $C^{\infty}(M)^{G}$ we have

$$
\Phi_{g}^{*}\left(L_{X} f\right)=L_{\Phi_{g}^{*} X}\left(\Phi_{g}^{*}(f)\right)=L_{X} f .
$$

$X$ is a derivation, because it is clearly a real linear mapping of $C^{\infty}(M)^{G}$ into itself, and it satisfies Leibniz' rule, since for $f_{1}, f_{2} \in C^{\infty}(M)^{G}$ we have

$$
X\left(f_{1} f_{2}\right)=L_{X}\left(f_{1} f_{2}\right)=\left(L_{X} f_{1}\right) f_{2}+f_{1}\left(L_{X} f_{2}\right)=X\left(f_{1}\right) f_{2}+f_{1} X\left(f_{2}\right) .
$$

For each $m \in M$ let $\varphi_{m}: I_{m} \subseteq \mathbb{R} \rightarrow M: t \mapsto \varphi_{t}(m)$ be a maximal integral curve of the $G$-invariant vector field $X$ on $M$, which starts at $m$. Here $I_{m}$ is an open interval containing 0 . Let

$$
\mathcal{D}=\amalg_{m \in M} I_{m}=\left\{(t, m) \in \mathbb{R} \times M \mid t \in I_{m} \text { for each } m \in M\right\} .
$$


Then $\mathcal{D}$ is an open subset of $\mathbb{R} \times M$, which is the domain of the local flow $\varphi: \mathcal{D} \subseteq \mathbb{R} \times M \rightarrow M$ of the vector field $X$, namely,

1) For each $m \in M$ we have $\varphi_{0}(m)=m$;

$2)$ if $(t, m) \in \mathcal{D},\left(s, \varphi_{t}(m)\right) \in \mathcal{D}$, and $(s+t, m) \in \mathcal{D}$, then $\varphi_{s+t}(m)=$ $\varphi_{s}\left(\varphi_{t}(m)\right)$.

For each $m \in M$ there is an open neighborhood $U_{m}$ of $m$ in $M$ such that the map $\mathcal{D}_{U_{m}} \subseteq \mathbb{R} \rightarrow C^{\infty}\left(U_{m}, M\right): t \mapsto \varphi_{t}$, where $\mathcal{D}_{U_{m}}=\left\{t \in \mathbb{R} \mid\left(t, m^{\prime}\right) \in\right.$ $\mathcal{D}$ for every $\left.m^{\prime} \in U_{m}\right\}$, is a local one parameter group of local diffeomorphisms of $M$.

Lemma 6.1.2 $X$ is a $G$-invariant vector field on $M$ if and only if

$$
\left(\Phi_{g} \circ \varphi_{t}\right)(m)=\left(\varphi_{t} \circ \Phi_{g}\right)(m)
$$

for every $(t, m) \in \mathcal{D}$.

Proof. If equation (25) holds, then differentiating both sides with respect to $t$ and then evaluating at $t=0$ gives the second equality below

$$
\begin{aligned}
T_{m} \Phi_{g} X(m) & =\left.T_{m} \Phi_{g} \frac{\mathrm{d}}{\mathrm{d} t}\right|_{t=0} ^{\varphi_{t}(m),} \quad \begin{array}{l}
\text { since } t \mapsto \varphi_{t}(m) \text { is an integral curve of } X \\
\text { starting at } m .
\end{array} \\
& =\left.\frac{\mathrm{d}}{\mathrm{d} t}\right|_{t=0} \varphi_{t}\left(\Phi_{g}(m)\right)=X\left(\Phi_{g}(m)\right) .
\end{aligned}
$$

Consequently, $\Phi_{g}^{*} X=X$, that is, the vector field $X$ is $G$-invariant. To prove the converse, note that $t \mapsto \varphi_{t}\left(\Phi_{g}(m)\right)$ is an integral curve of $X$ starting at $\Phi_{g}(m)$. We now show that $t \mapsto \Phi_{g}\left(\varphi_{t}(m)\right)$ is also an integral curve of $X$ starting at $\Phi_{g}(m)$. We have

$$
\begin{aligned}
\frac{\mathrm{d}}{\mathrm{d} t}\left(\Phi_{g} \circ \varphi_{t}\right)(m) & =T_{\varphi_{t}(m)} \Phi_{g}\left(\frac{\mathrm{d}}{\mathrm{d} t} \varphi_{t}(m)\right) \\
& =T_{\varphi_{t}(m)} \Phi_{g}\left(X\left(\varphi_{t}(m)\right)\right), \quad \text { since } t \mapsto \varphi_{t}(m) \text { is an integral } \\
& =X\left(\Phi_{g}\left(\varphi_{t}(m)\right)\right), \quad \text { since } X \text { is a } G \text {-invariant vector field } \\
& =X\left(\left(\Phi_{g} \circ \varphi_{t}\right)(m)\right) .
\end{aligned}
$$

Since maximal integral curves of the vector field $X$ starting at $\Phi_{g}(m)$ are unique, we get $\left(\Phi_{g} \circ \varphi_{t}\right)(m)=\left(\varphi_{t} \circ \Phi_{g}\right)(m)$ for every $(t, m) \in \mathcal{D}$.

From equation (25) it follows that $\varphi_{t}(G \cdot m)=G \cdot \varphi_{t}(m)$ for every $(t, m) \in \mathcal{D}$. Hence $\varphi_{t}$ induces the map

$$
\bar{\varphi}_{t}: \bar{M} \rightarrow \bar{M}: \bar{m}=G \cdot m \mapsto G \cdot \varphi_{t}(m)=\overline{\varphi_{t}(m)} .
$$

Lemma 6.1.3 For every $(t, m) \in \mathcal{D}$ we have

$$
\bar{\varphi}_{t} \circ \pi=\pi \circ \varphi_{t}
$$


Proof. Equation (27) follows from equation (26) because

$$
\bar{\varphi}_{t}(\pi(m))=\bar{\varphi}_{t}(\bar{m})=\overline{\varphi_{t}(m)}=\pi\left(\varphi_{t}(m)\right),
$$

for every $(t, m) \in \mathcal{D}$.

Let $X$ be a $G$-invariant vector field on $M$. Let

$$
\bar{X}: C^{\infty}(\bar{M}) \rightarrow C^{\infty}(\bar{M}): \bar{f} \mapsto \overline{X\left(\pi^{*} \bar{f}\right)},
$$

that is, $\pi^{*}(\bar{X}(\bar{f}))=X\left(\pi^{*} \bar{f}\right)$, for every $\bar{f} \in C^{\infty}(M)$. We can rewrite the preceding equation as

$$
T \pi \circ X=\bar{X} \circ \pi .
$$

Lemma 6.1.4 $\bar{X}$ is a derivation of $C^{\infty}(\bar{M})$.

Proof. $\bar{X}$ is a real linear mapping of $C^{\infty}(\bar{M})$ into itself, for if $\alpha, \beta \in \mathbb{R}$ and $\bar{f}_{1}$, $\bar{f}_{2} \in C^{\infty}(\bar{M})$ then

$$
\begin{aligned}
\bar{X}\left(\alpha \bar{f}_{1}\right. & \left.+\beta \bar{f}_{2}\right)=\overline{X\left(\pi^{*}\left(\alpha \bar{f}_{1}+\beta \bar{f}_{2}\right)\right)}, \quad \text { by definition of } \bar{X} \\
& =\overline{X\left(\pi^{*}\left(\alpha \bar{f}_{1}\right)+\pi^{*}\left(\beta \bar{f}_{2}\right)\right)} \\
& =\alpha \overline{X\left(\pi^{*}\left(\bar{f}_{1}\right)\right)}+\beta \overline{X\left(\pi^{*}\left(\bar{f}_{2}\right)\right)}, \text { since } X \text { is a derivation of } C^{\infty}(M)^{G} \\
& =\alpha \bar{X}\left(\bar{f}_{1}\right)+\beta \bar{X}\left(\bar{f}_{2}\right) .
\end{aligned}
$$

Also $\bar{X}$ satisfies Leibniz' rule, for

$$
\begin{aligned}
\bar{X}\left(\bar{f}_{1} \bar{f}_{2}\right) & =\overline{X\left(\pi^{*}\left(\bar{f}_{1} \bar{f}_{2}\right)\right.}=\overline{X\left(\pi^{*} \bar{f}_{1} \pi^{*} \bar{f}_{2}\right)} \\
& =\overline{X\left(\pi^{*} \bar{f}_{1}\right)} \overline{\pi^{*} \bar{f}_{2}}+\overline{\pi^{*} \bar{f}_{1}} \overline{X\left(\pi^{*} \bar{f}_{2}\right)} \\
& =\bar{X}\left(\bar{f}_{1}\right) \bar{f}_{2}+\bar{f}_{1} \bar{X}\left(\bar{f}_{2}\right) .
\end{aligned}
$$

Proposition 6.1.5 Let $\overline{\mathcal{D}}=\left\{(t, \bar{m}) \in \mathbb{R} \times \bar{M} \mid t \in \mathcal{D}_{m} \& \bar{m} \in \pi\left(\mathcal{D}_{t}\right)\right\}$ with $\mathcal{D}_{t}=\{m \in M \mid(t, m) \in \mathcal{D}\}$ and $\mathcal{D}_{m}=\{t \in \mathbb{R} \mid(t, m) \in \mathcal{D}\}$. Then the curve

$$
\bar{c}: \overline{\mathcal{D}}_{\bar{m}}=\mathcal{D}_{m} \subseteq \mathbb{R} \rightarrow \bar{M}: \bar{m} \mapsto \bar{\varphi}_{t}(\bar{m})
$$

is a maximal integral curve of the derivation $\bar{X}$ of $C^{\infty}(\bar{M})$ starting at $\bar{m} \in \bar{M}$.

Proof. Let $c: \mathcal{D}_{m} \subseteq \mathbb{R} \rightarrow M: t \mapsto \varphi_{t}(m)$ be a maximal integral curve of the $G$-invariant vector field $X$ on $M$. Then

$$
T c(t)=\left(X_{\circ} c\right)(t) \quad \text { for every } t \in \mathcal{D}_{m} \text { and every } m \in M .
$$

Since $\bar{c}=\pi_{*} c$ we get

$$
\begin{aligned}
T \bar{c}(t) & =T\left(\pi_{*} c\right)(t)=\left(T \pi_{*}\right)(T c)(t) \\
& \left.=\left(T \pi_{*}\right)(X \circ c)\right)(t), \quad \text { using (30) } \\
& =\bar{X}\left(\pi_{*} c\right)(t), \quad \text { using (29) } \\
& =(\bar{X} \circ \bar{c})(t) .
\end{aligned}
$$


Thus $\bar{c}$ is an integral curve of the derivation $\bar{X}$, which is maximal because by equation (29) the derivations $X$ on $C^{\infty}(M)^{G}$ and $\bar{X}$ on $C^{\infty}(\bar{M})$ are $\pi$-related and their local flows satisfy equation (28).

Corollary 6.1.5A The map

$$
\bar{\varphi}: \overline{\mathcal{D}} \subseteq \mathbb{R} \times \bar{M} \rightarrow \bar{M}:(t, \bar{m}) \mapsto \bar{\varphi}_{t}(\bar{m})
$$

is the local flow of the derivation $\bar{X}$ on $C^{\infty}(\bar{M})$.

Proof. This follows immediately from proposition 6.1.5.

Corollary 6.1.5B The derivation $\bar{X}$ on $C^{\infty}(\bar{M})$ is a vector field on $\bar{M}$.

Proof. Since $\mathcal{D}_{t}$ is an open subset of $M$ for all $t \in \mathbb{R}$ and the $G$-orbit map $\pi$ is an open map, we get $\overline{\mathcal{D}}_{t}=\pi\left(\mathcal{D}_{t}\right)$ is an open subset of $\bar{M}$. Also $\mathcal{D}_{m}$ is a open interval containing 0 for all $m \in M$, which implies $\overline{\mathcal{D}}_{\bar{m}}$ is an open interval containing 0 for all $\bar{m} \in \bar{M}$, since $\overline{\mathcal{D}}_{\bar{m}}=\mathcal{D}_{m}$. Thus every maximal integral curve of $\bar{X}$ is defined on an open interval in $\mathbb{R}$ that contains 0 and is also unique since every integral curve of $X$ is unique and the derivations $X$ and $\bar{X}$ are $\pi$-related. Hence by proposition 3.3.1 the derivation $\bar{X}$ on $\left(\bar{M}, C^{\infty}(\bar{M})\right)$ is a vector field.

\subsection{The differentiable space $\bar{M}$}

In this subsection we show that the $G$-orbit space $\left(\bar{M}, C^{\infty}(\bar{M})\right)$ is a differentiable space in the sense of Smith.

Lemma 6.2.1 The surjective continuous $G$-orbit mapping $\pi: M \rightarrow \bar{M}$ at every $\bar{m} \in \bar{M}$ has a continuous local section, that is, there is an open neighborhood $\bar{U}$ of $\bar{m}$ in $\bar{M}$ and a continuous mapping $\sigma_{\bar{U}}: \bar{U} \subseteq \bar{M} \rightarrow M$ such that $\pi_{\circ} \sigma_{\bar{U}}=\mathrm{id}_{\bar{U}}$.

Proof. Let $\bar{m} \in \bar{M}$. Suppose that $\widetilde{U}$ is an open subset of $M$ containing $m \in \pi^{-1}(\bar{m})$. Since the $G$ action on $M$ is proper, it has a slice $S_{m}$ at $m$. Let $U$ be an open neighborhood of $m$, which is contained in $\widetilde{U}$, such that its closure $\operatorname{cl}(U)$ is compact. Then the mapping $\left.\pi\right|_{\operatorname{cl}(U) \cap S_{m}}$ is a continuous bijection onto $\pi(\operatorname{cl}(U))$, which is a compact subset of the Hausdorff topological space $\bar{M}$. Hence the map $\left.\pi\right|_{\mathrm{cl}(U) \cap S_{m}}$ is a homeomorphism of $U \cap S_{m}$ onto the open neighborhood $\bar{U}=\pi\left(U \cap S_{m}\right)$ of $\bar{m}=\pi(m)$. Let $\left.\sigma\right|_{\bar{U}}=\left.\left(\pi^{-1}\right)\right|_{\bar{U}}: \bar{U} \subseteq \bar{M} \rightarrow M$. Since $\pi_{\circ} \sigma_{\bar{U}}=\mathrm{id}_{\bar{U}}$ by construction, $\sigma_{\bar{U}}$ is a continuous local section of the mapping $\pi$.

Theorem 6.2.2 The differential space $\left(\bar{M}, C^{\infty}(\bar{M})\right)$ is a differentiable space in the sense of Smith.

To prove theorem 6.2.2 we will need some preliminary results.

Lemma 6.2.3 If $\bar{c} \in C^{0}(\mathbb{R}, \bar{M})$, the set of continuous maps from $\mathbb{R}$ to the $G$ orbit space $\bar{M}$, then there is $c \in C^{0}(\mathbb{R}, M)$, which covers $\bar{c}$, that is, $\bar{c}=\pi_{\circ} c$. 
Proof. Let $\bar{c} \in C^{0}(\mathbb{R}, \bar{M})$ be given. Let $\mathcal{T}^{+}$be the set of all $t \in \mathbb{R}_{>0}$ such that there is a continuous curve $c:[0, t) \rightarrow M$, which covers the given continuous curve $\left.\bar{c}\right|_{[0, t)}$. Let $c(0) \in \pi^{-1}(\bar{c}(0))$. By lemma 6.2.1 there is an open neighborhood $\bar{U}_{\bar{c}(0)}$ of $\bar{c}(0)$ in $\bar{M}$ and a local section $\sigma_{\bar{U}_{\bar{c}(0)}}: \bar{U}_{\bar{c}(0)} \subseteq \bar{M} \rightarrow M$ of the $G$-orbit map $\pi: M \rightarrow \bar{M}$ such that $\sigma_{\bar{U}_{\bar{c}(0)}}(\bar{c}(0))=c(0)$. Since $\bar{U}_{\bar{c}(0)}$ is an open neighborhood of $\bar{c}(0)$, there is an $\varepsilon_{\bar{c}(0)}>0$ such that $\bar{c}\left(\left[0, \varepsilon_{\bar{c}(0)}\right)\right) \subseteq \bar{U}_{\bar{c}(0)}$. On $\left[0, \varepsilon_{\bar{c}(0)}\right)$ let $c=\sigma_{\bar{U}_{\bar{c}(0)}} \circ \bar{c}$. Then $c$ is a continuous curve starting at $c(0)$ and defined on $\left[0, \varepsilon_{\bar{c}(0)}\right)$, which covers $\bar{c}$ on $\left[0, \varepsilon_{\bar{c}(0)}\right)$, since $\pi_{\circ} c=\pi \circ\left(\sigma_{\bar{U}_{\bar{c}(0)}}{ }^{\circ} c\right)=c$ on $\left[0, \varepsilon_{\bar{c}(0)}\right)$. This shows that $\varepsilon_{\bar{c}(0)} \in \mathcal{T}^{+}$. Since $\mathcal{T}^{+}$is nonempty, it has a supremum $T^{+}$. Suppose that $T^{+}<\infty$. By lemma 6.2.1 there is an open neighborhood $\bar{U}_{\bar{c}\left(T^{+}\right)}$of $\bar{c}\left(T^{+}\right)$in $\bar{M}$ and a continuous local section $\sigma_{\bar{U}_{\bar{c}\left(T^{+}\right)}}: \bar{U}_{\bar{c}\left(T^{+}\right)} \subseteq \bar{M} \rightarrow M$ of the $G$-orbit map $\pi$ such that for some $\tilde{t} \in \mathcal{T}^{+}$we have $\bar{c}(\widetilde{t}) \in \bar{U}_{\bar{c}\left(T^{+}\right)}$. Since $\widetilde{t} \in \mathcal{T}^{+}$, there is a continuous curve $c$ on $M$ defined on $[0, \widetilde{t})$, which covers $\left.\bar{c}\right|_{[0, \widetilde{t})}$. So $\bar{c}(\widetilde{t})=\pi(c(\widetilde{t}))$. Define $\widetilde{c}=\sigma_{\bar{U}_{\bar{c}\left(T^{+}\right)}} \circ \bar{c}$. Then $\widetilde{c}$ is a continuous curve on $\left[0, T^{+}+\varepsilon_{\bar{c}\left(T^{+}\right)}\right)$, where $\varepsilon_{\bar{c}\left(T^{+}\right)}>0$ is chosen so that $\bar{c}\left(\left[0, T^{+}+\varepsilon_{\bar{c}\left(T^{+}\right)}\right)\right) \subseteq \bar{U}_{\bar{c}\left(T^{+}\right)}$. Moreover, $\left.\widetilde{c}\right|_{[0, \widetilde{t})}=\left.c\right|_{[0, \widetilde{t})}$. This contradicts the definition of $T^{+}$. Hence $T^{+}=\infty$. A similar agrument shows that $\mathcal{T}^{-}$, the set of all $t \in \mathbb{R}_{<0}$ such that there is a continuous curve $c:(-t, 0] \subseteq \mathbb{R} \rightarrow M$, which covers the given continuous curve $\bar{c}$ on $(-t, 0]$, is nonempty and does not have a finite infimum. Consequently, for a given continuous curve $\bar{c}: \mathbb{R} \rightarrow \bar{M}$ there is a continuous curve $c: \mathbb{R} \rightarrow M$, which covers $\bar{c}$.

In our context we have

$$
\Gamma_{0} C^{\infty}(M)^{G}=\left\{c \in C^{0}(\mathbb{R}, M) \mid f_{\circ} c \in C^{\infty}(\mathbb{R}) \text { for all } f \in C^{\infty}(M)^{G}\right\}
$$

and

$$
\Phi_{0} \Gamma_{0} C^{\infty}(\bar{M})=\left\{\bar{f} \in C^{0}(\bar{M}) \mid \bar{f}_{\circ} \bar{c} \in C^{\infty}(\mathbb{R}) \text { for all } \bar{c} \in \Gamma_{0} C^{\infty}(\bar{M})\right\},
$$

see equation (44).

Lemma 6.2.4 We have

$$
\pi_{*}\left(\Gamma_{0} C^{\infty}(M)^{G}\right)=\Gamma_{0} C^{\infty}(\bar{M}),
$$

that is, if $\bar{c} \in \Gamma_{0} C^{\infty}(\bar{M})$, then there is a $c \in \Gamma_{0} C^{\infty}(M)^{G}$ such that $\bar{c}=\pi_{*}(c)=$ $\pi_{\circ} c$ and conversely, if $c \in \Gamma_{0} C^{\infty}(M)^{G}$, then $\pi_{*}(c)=\bar{c} \in \Gamma_{0} C^{\infty}(\bar{M})$.

Proof. Suppose that $\bar{c} \in \Gamma_{0} C^{\infty}(\bar{M}) \subseteq C^{0}(\bar{M})$. Then $\bar{f}_{\circ} \bar{c} \in C^{\infty}(\mathbb{R})$ for every $\bar{f} \in C^{\infty}(\bar{M})$. By lemma 6.2.3 there is $c \in C^{0}(\mathbb{R}, M)$ such that $\bar{c}=\pi_{*}(c)$. Hence

$$
C^{\infty}(\mathbb{R}) \ni \bar{f} \circ \bar{c}=(\bar{f} \circ \pi) \circ c=\left(\pi^{*} \bar{f}\right) \circ c=f \circ c
$$

for every $f \in C^{\infty}(M)^{G}$, since $C^{\infty}(M)^{G}=\pi^{*}\left(C^{\infty}(\bar{M})\right)$. In other words, $c \in$ $\Gamma_{0} C^{\infty}(M)^{G}$. So $\bar{c}=\pi_{*}(c) \in \pi_{*}\left(\Gamma_{0} C^{\infty}(M)^{G}\right)$. Consequently, $\Gamma_{0} C^{\infty}(\bar{M}) \subseteq$ $\pi_{*}\left(\Gamma_{0} C^{\infty}(M)^{G}\right)$. To verify the reverse inclusion suppose $\bar{c} \in \pi_{*}\left(\Gamma_{0} C^{\infty}(M)^{G}\right)$. 
Then there is a $c \in \Gamma_{0} C^{\infty}(M)^{G}$ such that $\bar{c}=\pi_{*}(c)$. Suppose that $\bar{f} \in C^{\infty}(\bar{M})$. Then $f=\pi^{*} \bar{f} \in C^{\infty}(M)^{G}$. Now

$$
C^{\infty}(\mathbb{R}) \ni f \circ c=\left(\pi^{*} \bar{f}\right) \circ c=\bar{f}_{\circ}(\pi \circ c)=\bar{f} \circ \bar{c},
$$

for every $f \in C^{\infty}(M)^{G}$ and hence for all $\bar{f} \in C^{\infty}(\bar{M})$, since $c \in \Gamma_{0} C^{\infty}(M)^{G}$ and $\pi^{*}\left(C^{\infty}(\bar{M})\right)=C^{\infty}(M)^{G}$. Thus $\bar{c} \in \Gamma_{0} C^{\infty}(\bar{M})$. So we have shown that $\Gamma_{0} C^{\infty}(M)^{G} \subseteq \Gamma_{0} C^{\infty}(\bar{M})$. This verifies (31).

Claim 6.2.5 The differential structure $C^{\infty}(\bar{M})$ is continuously reflexive, that is

$$
C^{\infty}(\bar{M})=\Phi_{0} \Gamma_{0}\left(C^{\infty}(\bar{M})\right)
$$

To prove claim 6.2 .5 we will need some additional results.

Lemma 6.2.6 We have

$$
\Gamma_{0} C^{\infty}(M) \subseteq \Gamma_{0} C^{\infty}(M)^{G} .
$$

Proof. Let $c \in \Gamma_{0} C^{\infty}(M)$. Then $c \in C^{0}(\mathbb{R}, M)$ and for every $f \in C^{\infty}(M)$ we have $f_{\circ} c \in C^{\infty}(\mathbb{R})$. Thus $f_{\circ} c \in C^{\infty}(\mathbb{R})$ for every $f \in C^{\infty}(M)^{G}$ because $C^{\infty}(M)^{G} \subseteq C^{\infty}(M)$. So $c \in \Gamma_{0} C^{\infty}(M)^{G}$. This verifies (33).

Lemma 6.2.7 We have

$$
\Phi_{0} \Gamma_{0} C^{\infty}(M)^{G} \subseteq \Phi_{0} \Gamma_{0} C^{\infty}(M) .
$$

Proof. Let $f \in \Phi_{0} \Gamma_{0} C^{\infty}(M)^{G} \subseteq C^{0}(M)^{G}$. Then $f_{\circ} c \in C^{\infty}(\mathbb{R})$ for all $c \in$ $\Gamma_{0} C^{\infty}(M)^{G}$. Since $\Gamma_{0} C^{\infty}(M) \subseteq \Gamma_{0} C^{\infty}(M)^{G}$ by lemma 6.2 .6 , we obtain $f_{\circ} c \in$ $C^{\infty}(\mathbb{R})$ for all $c \in \Gamma_{0} C^{\infty}(M)$. In other words, $f \in \Phi_{0} \Gamma_{0} C^{\infty}(M)$. This verifies (34).

Proposition 6.2.8 $C^{\infty}(M)^{G}$ is continuously reflexive.

Proof. Using the fact that $C^{\infty}(M)^{G} \subseteq \Phi_{0} \Gamma_{0}\left(C^{\infty}(M)^{G}\right)$, we need only show that $\Phi_{0} \Gamma_{0}\left(C^{\infty}(M)^{G}\right) \subseteq C^{\infty}(M)^{G}$. Suppose that $f \in \Phi_{0} \Gamma_{0} C^{\infty}(M)^{G}$. Then $f \in C^{0}(M)^{G}$, that is, $f$ is a continuous $G$-invariant function on $M$. Since $\Phi_{0} \Gamma_{0} C(M)^{G} \subseteq \Phi_{0} \Gamma_{0} C^{\infty}(M)$ by lemma 6.2 .7 and $f \in \Phi_{0} \Gamma_{0} C^{\infty}(M)^{G}$ by hypothesis, we get $f \in \Phi_{0} \Gamma_{0} C^{\infty}(M)=C^{\infty}(M)$. The preceding equality follows since the differential structure $C^{\infty}(M)$ on the smooth manifold $M$ is continuously reflexive. So $f$ is a smooth function on $M$. Since $f$ is $G$-invariant, it follows that $f \in C^{\infty}(M)^{G}$. This shows that $\Phi_{0} \Gamma_{0} C^{\infty}(M)^{G} \subseteq C^{\infty}(M)^{G}$.

Lemma 6.2.9 We have

$$
\pi^{*}\left(\Phi_{0} \Gamma_{0} C^{\infty}(\bar{M})\right)=\Phi_{0} \Gamma_{0} C^{\infty}(M)^{G} .
$$

Proof. Suppose that $\bar{f} \in \Phi_{0} \Gamma_{0} C^{\infty}(\bar{M}) \subseteq C^{0}(\bar{M})$. Then for every $\bar{c} \in$ $\Gamma_{0} C^{\infty}(\bar{M})$ we have $\bar{f} \circ \bar{c} \in C^{\infty}(\mathbb{R})$. Since $\bar{\Gamma}_{0} C^{\infty}(\bar{M})=\pi_{*}\left(\Gamma_{0} C^{\infty}(M)^{G}\right)$ by lemma 6.2.4, there is $c \in \Gamma_{0} C^{\infty}(M)^{G}$ such that $\bar{c}=\pi \circ c$. So

$$
C^{\infty}(\mathbb{R}) \ni \bar{f} \circ \bar{c}=(\bar{f} \circ \pi) \circ c=\pi^{*} \bar{f} \circ c,
$$


for every $c \in \Gamma_{0} C^{\infty}(M)^{G}$. Thus $\pi^{*} \bar{f} \in \Phi_{0} \Gamma_{0} C^{\infty}(M)^{G}$. So we have shown $\pi^{*}\left(\Phi_{0} \Gamma_{0} C^{\infty}(\bar{M})\right) \subseteq \Phi_{0} \Gamma_{0} C^{\infty}(M)^{G}$. To verify the reverse inclusion let $f \in$ $\Phi_{0} \Gamma_{0} C^{\infty}(M)^{G} \subseteq C^{0}(M)^{G}$. Then $f_{\circ} c \in C^{\infty}(\mathbb{R})$ for every $c \in \Gamma_{0} C^{\infty}(M)^{G}$. Since $C^{0}(M)^{G}=\pi^{*}\left(C^{0}(\bar{M})\right)$, there is $\bar{f} \in C^{0}(\bar{M})$ such that $f=\pi^{*} \bar{f}$. So

$$
C^{\infty}(\mathbb{R}) \ni f \circ c=\pi^{*} \bar{f}_{\circ} c=\bar{f}_{\circ}(\pi \circ c),
$$

for every $c \in \Gamma_{0} C^{\infty}(M)^{G}$. By lemma 6.2.4, $\pi_{*}\left(\Gamma_{0} C^{\infty}(M)^{G}\right)=\Gamma_{0} C^{\infty}(\bar{M})$. Consequently, $\bar{f}_{\circ}(\pi \circ c)=\bar{f}_{\circ} \bar{c} \in C^{\infty}(\mathbb{R})$ for every $\bar{c} \in \Gamma_{0} C^{\infty}(\bar{M})$. So $\bar{f} \in$ $\Phi_{0} \Gamma_{0} C^{\infty}(\bar{M})$. In other words, $f \in \pi^{*}\left(\Phi_{0} \Gamma_{0} C^{\infty}(\bar{M})\right)$. Thus $\Phi_{0} \Gamma_{0} C^{\infty}(M)^{G} \subseteq$ $\pi^{*}\left(\Phi_{0} \Gamma_{0} C^{\infty}(\bar{M})\right)$. This verifies (35)).

Proof of claim 6.2.5 From lemma 6.2.9 it follows that we have $\pi^{*}\left(\Phi_{0} \Gamma_{0} C^{\infty}(\bar{M})\right)$ $=\Phi_{0} \Gamma_{0} C^{\infty}(M)^{G}$. But $C^{\infty}(M)^{G}$ is continuously reflexive by proposition 6.2.8. Thus

$$
\pi^{*}\left(\Phi_{0} \Gamma_{0} C^{\infty}(\bar{M})\right)=C^{\infty}(M)^{G}=\pi^{*}\left(C^{\infty}(\bar{M})\right) .
$$

Since the $G$-orbit mapping $\pi$ is surjective, the map

$$
\pi^{*}: C^{\infty}(\bar{M}) \rightarrow C^{\infty}(M)^{G}: \bar{f} \mapsto \pi^{*}(\bar{f})
$$

is injective. Hence $\Phi_{0} \Gamma_{0} C^{\infty}(\bar{M})=C^{\infty}(\bar{M})$, which verifies (32).

Proof of theorem 6.2.2 This follows from theorem 4.3, since the differential structure $C^{\infty}(\bar{M})$ is continuously reflexive by claim 6.2 .5 .

\subsection{Additional properties}

We now verify the extra hypotheses on a differentiable space so that the hypotheses of Smith's de Rham theorem, theorem 5.5.3, hold for the differentiable space $\left(\bar{M}, C^{\infty}(\bar{M})\right)$.

Claim 6.3.1 The subcartesian differential space $\left(\bar{M}, C^{\infty}(\bar{M})\right)$ is a locally differentiably contractible differentiable space.

Proof. Let $m \in M$. Since the $G$-action $\Phi$ on the smooth manifold $M$ is proper, there is a $G_{m}$-invariant slice $S$ to the $G$-action at $m$. Because the isotropy group $G_{m}=\left\{g \in G \mid \Phi_{g}(m)=m\right\}$ is compact, there is an open neighborhood $V_{m}$ of 0 in $T_{m} M$ and a diffeomorphism $\psi: V_{m} \subseteq T_{m} M \rightarrow U_{m} \subseteq M$ such that for every $g \in G_{m}$ and every $v_{m} \in V_{m}$ we have $T_{m} \psi\left(T_{m} \Phi_{g} v_{m}\right)=T_{m} \Phi_{g}\left(T_{m} \psi v_{m}\right)$. Let $\gamma_{m}$ be a $G_{m}$-invariant Euclidean inner product on $T_{m} M$ and let $B_{m}^{\varepsilon}$ be a $\gamma_{m}$ ball of radius $\varepsilon$ with center 0 contained in $V_{m}$. Let $Y_{m}$ be a linear vector field on $T_{m} M$ whose flow is $\varphi_{t}^{Y_{m}}\left(v_{m}\right)=\mathrm{e}^{-t} v_{m}$. Let $X_{m}=\frac{1}{\operatorname{vol} G_{m}} \int_{G_{m}}\left(T_{m} \Phi_{h}\right)^{*} Y_{m} \mathrm{~d} h$, where $\mathrm{d} h$ is a volume form on $G_{m}$, be the vector field on $T_{m} M$, which is the $G_{m}$ average of the pull back of $Y_{m}$ by the action $T_{m} \Phi_{h}$ of the $G_{m}$ action on $T_{m}$. Then $X_{m}$ is a linear $G_{m}$-invariant vector field on $T_{m} M$, whose flow $\varphi_{t}^{X_{m}}$ commutes with the $\varphi_{t}^{Y_{m}}$ of the vector field $Y_{m}$. Hence the flow of the $G_{m}$-invariant vector field $\mathcal{X}_{m}=\psi_{*} X_{m}$ on $U_{m}$ preserves the slice $\mathcal{S}=S \cap \psi\left(B_{m}^{\varepsilon}\right)$ and contracts it to the point $m$. The vector field $\mathcal{X}\left(\Phi_{g}(m)\right)=\left(\Phi_{g}\right)_{*} \mathcal{X}_{m}$ is $\Phi_{g}$-invariant on $G \cdot \mathcal{S}$. Its 
flow $\varphi_{t}^{\mathcal{X}}$ contracts $G \cdot \mathcal{S}$ to the $G$-orbit $G_{m}$ and commutes with the $G$ action $\Phi$. Let $\overline{\mathcal{S}}=\pi(G \cdot \mathcal{S})$. Then $\overline{\mathcal{S}}$ is an open neighborhood of $\bar{m}=\pi(m)$ in the quotient topology (and hence the differential space topology) of $\left(\bar{M}, C^{\infty}(\bar{M})\right)$. Let $\overline{\mathcal{X}}$ be the vector field on $\bar{M}$ induced by the $G$-invariant vector $\mathcal{X}$. Since $\varphi^{\overline{\mathcal{X}}} \circ \pi=\pi_{\circ} \varphi_{t}^{\mathcal{X}}$, the flow $\varphi^{\overline{\mathcal{X}}}$ is $\pi$-related to the flow $\varphi_{t}^{\mathcal{X}}$ of $\mathcal{X}$ on $M$, that is, $\varphi_{t}^{\overline{\mathcal{X}}} \circ \pi=\pi \circ \varphi_{t}^{\mathcal{X}}$. From the fact that $\varphi_{t}^{\mathcal{X}}$ smoothly contracts $G \cdot \mathcal{S}$ to the $G$-orbit $G \cdot m$, we determine that $\varphi_{t}^{\overline{\mathcal{X}}}$ smoothly contracts the open neighborhood $\overline{\mathcal{S}}$ of $\bar{m}$ to $\bar{m}$.

Let $\left(S, C^{\infty}(S)\right)$ be a locally closed (and hence paracompact) subcartesian space. Let $U$ be an open subset of $S$. The assignment

$$
U \mapsto C^{\infty}(U)=\left.C^{\infty}(S)\right|_{U}
$$

defines an abelian sheaf $\mathcal{S}$ of rings with a unit.

Lemma 6.3.2 The sheaf $\mathcal{S}$ is soft, that is, if $T$ is a closed subset of $S$ and $s \in C^{\infty}(T)=\left.C^{\infty}(S)\right|_{T}$, then there is a $\Sigma \in C^{\infty}(S)$ such that $\left.\Sigma\right|_{T}=s$.

Proof. Let $U_{0}=S \backslash T$ and let $\left\{U_{i}\right\}_{i \in I}$ be an open covering of $S$. Then $\left\{U_{i}\right\}_{j \in I \cup\{0\}}$ is an open covering of $S$. Since $S$ is paracompact, $\left\{U_{i}\right\}_{j \in I \cup\{0\}}$ has a locally finite subcovering $\mathcal{V}=\left\{U_{j}\right\}_{j \in J \subset(I \cup\{0\})}$, Let $\left\{\psi_{j}\right\}_{j \in J}$ be a partition of unity in $C^{\infty}(S)$ associated to $\mathcal{V}$. Then $\Sigma=\sum_{j \in J} \psi_{j} s$ is a smooth function on $S$, whose restriction to $T$ is $s$, because $\sum_{j \in J} \psi_{j}(p)=1$ for every $p \in T$. Hence the sheaf $\mathcal{S}$ is soft.

Theorem 6.3.3 The paracompact subcartesian space $\left(\bar{M}, C^{\infty}(\bar{M})\right)$, where $\bar{M}$ is the orbit space of a proper action of a connected Lie group on a smooth manifold $M$ is a locally closed differential space, which is locally differentiably contractible and whose sheaf $\mathcal{S}$ of smooth functions is soft.

Proof. This follows immediately from claim 6.3.1 and lemma 6.3.2.

\section{4 de Rham's theorem for $\left(\bar{M}, C^{\infty}(\bar{M})\right.$}

Using theorem 6.3.3 it follows that Smith's version of de Rham's theorem 5.5.3 holds for $\left(\bar{M}, C^{\infty}(\bar{M})\right)$, namely,

Theorem 6.4.1 The cohomology of the exterior differential algebra $(\Lambda(\bar{M}), \overline{\mathrm{d}})$ of exterior differential forms on $\left(\bar{M}, C^{\infty}(\bar{M})\right)$ is isomorphic to the subalgebra $\mathcal{A}$ of the exterior differential algebra $\left(\Lambda(M)^{G}\right.$, d) of $G$-invariant differential forms on $M$ generated by differential 1-forms of smooth $G$-invariant functions on $M$ with coefficients which are smooth $G$-invariant functions.

The following example shows that the differential forms in Smith's version of de Rham's theorem [13] are not the basic forms used in Koszul's formulation of de Rham's theorem, see [8] and also Karshon and Watts [7].

Example 6.4.2 We follow Smith [13, p.133-134]. Consider the $\mathbb{Z}_{2}=\{-1,1\}$ action on $\mathbb{R}^{2}$ with coordinates $(x, y)$ generated by

$$
\zeta: \mathbb{R}^{2} \rightarrow \mathbb{R}^{2}:(x, y) \mapsto(-x,-y) .
$$


Since $\mathbb{Z}_{2}$ is finite, the $\mathbb{Z}_{2}$ action on $\mathbb{R}^{2}$ is proper. It is straightforward to check that the algebra of $\mathbb{Z}_{2}$ invariant polynomials on $\mathbb{R}^{2}$ is generated by

$$
\pi_{1}=x^{2}, \pi_{2}=x y, \text { and } \pi_{3}=y^{2} .
$$

They are subject to the relation

$$
\pi_{3}^{2}=\pi_{1} \pi_{2}, \pi_{1} \geq 0 \& \pi_{2} \geq 0,
$$

which defines the orbit space $\mathbb{R}^{2} / \mathbb{Z}_{2}$.

Consider the differential 1-form $\omega=x \mathrm{~d} y-y \mathrm{~d} x$ on $\mathbb{R}^{2}$, which is clearly $\mathbb{Z}_{2}$ invariant. Since the Lie algebra $\mathbb{Z}_{2}$ of $\mathbb{Z}_{2}$ is $\{0\}$, we have $L_{X_{\xi}} \omega=0$ for every $\xi \in \mathbb{Z}_{2}$. So $\omega$ is a basic 1 -form on $\mathbb{R}^{2}$ and hence induces a 1 -form $\bar{\omega}$ on $\mathbb{R}^{2} / \mathbb{Z}_{2}$.

We now show that $\omega$ is not a sum of 1 -forms of the form $f_{0} \mathrm{~d} f_{1}$, where $f_{0}$ is a smooth $\mathbb{Z}_{2}$ invariant function on $\mathbb{R}^{2}$ and $f_{1}$ is a $\mathbb{Z}_{2}$ invariant polynomial. Using polar coordinates $(\theta, r)$ on $\mathbb{R}^{2}$, we see that $\omega=r^{2} \mathrm{~d} \theta$. Let $\gamma_{R}$ be the circle $x^{2}+y^{2}=R^{2}$ in $\mathbb{R}^{2}$. Then

$$
\int_{\gamma_{R}} \omega=2 \pi R^{2}=\mathrm{O}\left(R^{2}\right)
$$

Since the invariant polynomials $\pi_{j}$ are quadratic in $x$ and $y$, all the odd order terms in the Taylor expansion of $f_{i}$ at $(0,0)$ to fourth order vanish. Hence

$$
f_{i}(x, y)=a_{i}+b_{i} x^{2}+c_{i}(x y)+d_{i} y^{2}+\mathrm{O}\left(\left(x^{2}+y^{2}\right)^{2}\right),
$$

where $a_{i}, b_{i}, c_{i}$, and $d_{i} \in \mathbb{R}$. Thus

$$
\int_{\gamma_{R}} f_{0} \mathrm{~d} f_{1}=b_{0} c_{1} \int_{\gamma_{R}} x^{2} \mathrm{~d}(x y)+d_{0} c_{1} \int_{\gamma_{R}} y^{2} \mathrm{~d}(x y)+\mathrm{O}\left(\int_{\gamma_{R}} r^{4}\right),
$$

since a straightforward calculation shows that the integral $\int_{\gamma_{R}}$ of the 1 forms $x \mathrm{~d} x, \mathrm{~d}(x y), y \mathrm{~d} y, x^{3} \mathrm{~d} x, x^{2} y \mathrm{~d} y,(x y) \mathrm{d}(x y), y^{2} \mathrm{~d} x$, and $y^{3} \mathrm{~d} y$ vanish. But

$$
\int_{\gamma_{R}} x^{2} \mathrm{~d}(x y)=-R^{4} \int_{0}^{2 \pi} \cos ^{2} \theta \mathrm{d} \theta+R^{4} \int_{0}^{2 \pi} \cos ^{4} \theta \mathrm{d} \theta=C R^{4},
$$

where $C \neq 0$ and $\int_{\gamma_{R}} y^{2} \mathrm{~d}(x y)=-\int_{\gamma_{R}} x^{2} \mathrm{~d}(x y)$. Hence

$$
\int_{\gamma_{R}} f_{0} \mathrm{~d} f_{1}=c_{1} C\left(b_{0}-d_{0}\right) R^{4}+\mathrm{O}\left(R^{5}\right)=\mathrm{O}\left(R^{4}\right),
$$

which contradicts equation (39).

Thus the exterior algebra of basic forms on $\mathbb{R}^{2}$ differs from the exterior algebra generated by the exterior derivative of $\mathbb{Z}_{2}$ invariant functions with coefficients which are smooth $\mathbb{Z}_{2}$-invariant functions. However, Koszul's version of de Rham's theorem and Smith's version yield the same real cohomology groups for $\mathbb{R}^{2} / \mathbb{Z}_{2}$, because they both give Cech cohomology with values in the sheaf $R$ of locally constant real valued functions on $\mathbb{R}^{2} / \mathbb{Z}_{2}$, since $\mathbb{R}^{2}$ is connected. 


\section{References}

[1] N. Aronszajn, Subcartesian and subRiemannian spaces, Notices American Mathematical Society 14 (1967) 111.

[2] A. Batubenge, P. Iglesias-Zenmour, Y. Karshon and J. Watts, Diffeological, Frölicher, and differential spaces, arxiv:math.DG.1712.0457v1

[3] R. Cushman and J. Śniatycki, On subcartesian spaces Leibniz' rule implies the chain rule, Canad. Math. Bull. (to appear).

[4] R. Cushman and J. Śniatycki, Differential structure of orbit spaces, Canad. Math. J. 54 (2001) 715-755.

[5] R.H. Cushman and L.M. Bates, Global aspects of classical integrable systems, second edition, Birkhäuser, Basel, 2015.

[6] J.J. Duistermaat, Dynamical systems with symmetry, University of Utrecht, www.staff.science.uu.nl/-kolk0101/homepageHD/sym.pdf

[7] Y. Karshon and J. Watts, Basic forms and orbit spaces, SIGMA 12 (2016) 02619 pages.

[8] J.L. Koszul, Sur certains groupes de transformations de Lie. Géométrie différentielle. Colloques Internationaux du Centre National de la Recherche Scientifique, Strasbourg, 1953, pp. 137-141. Centre National de la Recherche Scientifique, Paris, 1953.

[9] O.V. Lukina, F. Takens, and H.W. Broer, Global properties of integrable Hamiltonian systems, Reg. and chaotic dyn. 13 (2008) 602-644.

[10] R. Palais, On the existence of slices for actions of noncompact Lie groups, Annals of Mathematics 73 (1961) 295-323.

[11] R. Sikorski, Abstract covariant derivative, Colloq. Math. 18 (1967) 251272 .

[12] R. Sikorski, Wstęp do geometrii róźniczkowej [Introduction to differential geometry], Państwowe Wydawnictwo Naukowe, Warsaw, 1972.

[13] J.W. Smith, On the de Rham theorem for general spaces, Tohoku Math. Journ. 18 (1966) 115-137.

[14] J. Śniatycki, Differential geometry of singular spaces and reduction of symmetry, Cambridge University Press, Cambridge, UK, 2013.

[15] P. Walczak, A theorem on diffeomorphisms in the category of differential spaces. Bull. Acad. Polon. Sci. Sér. Sci. Math. Astronom. Phys. 21 (1973) 325-329. 